\title{
An $\mathcal{N}=1$ duality cascade from a deformation of $\mathcal{N}=4$ SUSY Yang-Mills theory
}

\author{
Timothy J. Hollowood ${ }^{a}$ and S. Prem Kumar ${ }^{a, b}$ \\ ${ }^{a}$ Department of Physics, \\ University of Wales Swansea, \\ Swansea, SA2 8PP, UK. \\ ${ }^{b} D A M T P$, \\ University of Cambridge, \\ Wilberforce Road, \\ Cambridge, CBЗ 0WA, UK. \\ E-mail: t.hollowood@swan.ac.uk, s.p.kumar@damtp.cam.ac.uk
}

\begin{abstract}
We study relevant deformations of an $\mathcal{N}=1$ superconformal theory which is an exactly marginal deformation of $U(N) \mathcal{N}=4$ SUSY Yang-Mills. The resulting theory has a classical Higgs branch that is a complex deformation of the orbifold $\mathbf{C}^{3} / \mathbf{Z}_{n} \times \mathbf{Z}_{n}$ that is a non-compact Calabi-Yau space with isolated conifold singularities. At these singular points in moduli space the theory exhibits a duality cascade and flows to a confining theory with a mass gap. By exactly solving the corresponding holomorphic matrix model we compute the exact quantum superpotential generated at the end of the duality cascade and calculate precisely how quantum effects deform the classical moduli space by replacing the conifold singularities with three-cycles of finite size. Locally the structure is that of the deformed conifold, but the global geometry is different. This desingularized quantum deformed geometry is the moduli space of probe D3-branes at the end of a duality cascade realized on the worldvolume of (fractional) D3-branes placed at the isolated conifold singularities in the deformation of the orbifold $\mathbf{C}^{3} / \mathbf{Z}_{n} \times \mathbf{Z}_{n}$ with discrete torsion.
\end{abstract}




\section{Introduction}

Gauge theories with $\mathcal{N}=1$ supersymmetry (SUSY) in four dimensions are known to exhibit rich and beautiful dynamics. The phenomenon of Seiberg duality plays a central role in much of this [1]. One of the most striking examples of the rich physics of $\mathcal{N}=1$ SUSY theories is provided by the duality cascade phenomenon uncovered by Klebanov and Strassler [2]. They investigated an $\mathcal{N}=1$ gauge theory with $S U(M+N) \times S U(N)$ gauge group and matter fields in the bi-fundamental representation which, upon renormalization group $(\mathrm{RG})$ flow, undergoes a succession of Seiberg dualities or a duality cascade. The number of colors in each factor drops repeatedly by $M$ units until finally in the infrared (IR) we are left with a pure $\mathcal{N}=1$ SUSY Yang-Mills theory that confines and produces a mass gap. ${ }^{1}$ In the ultraviolet (UV), however, these theories are not asymptotically free and hence are not well-defined quantum field theories at all energy scales.

In this article, we study the phenomenon of a duality cascade within a four dimensional UV-finite $\mathcal{N}=1$ SUSY field theory. Our study was motivated by the program initiated by Berenstein in [4-6]. The theory in question is obtained from $\mathcal{N}=4$ SUSY Yang-Mills theory with $U(N)$ gauge group by deformations involving exactly marginal and relevant operators. Thus a UV-completion of the KlebanovStrassler cascade can be achieved within a well-defined quantum field theory with gauge group of finite rank. Indeed, the theory we study has discrete branches where it flows, after a duality cascade, to an IR $\mathcal{N}=1$ gauge theory with a mass gap. This IR theory (more precisely, its holomorphic sector) is identical to the theory studied in [7] which is quite similar to $\mathcal{N}=1^{*}$ theory $[8,9]$. In the D-brane language, the theories that we study are realized on the worldvolume of D3-branes placed at isolated conifold singularities of a Calabi-Yau threefold which, in this case, is a complex deformation of the orbifold $\mathbf{C}^{3} / \mathbf{Z}_{n} \times \mathbf{Z}_{n}$. The endpoint of the duality cascade in the field theory has a geometric dual description where the D-branes have disappeared and the CalabiYau geometry is deformed and desingularized by the appearance of three-cycles of finite size. One of our aims is to obtain the precise algebraic description of this quantum deformed geometry with deformation parameters determined exactly in terms of the parameters of the gauge theory. Although near the singularities its local structure is that of the deformed conifold, the global structure is very different. From this deformed geometry we will also understand how the theory of [2] emerges as a limiting case when one of the finite three-cycles in the geometry is sent to infinity and we are left with a deformed conifold geometry. The main tool that we will employ to understand this deformed geometry (which is also the moduli space of probe D-branes at the end of the cascade) and the gauge theory superpotential,

\footnotetext{
${ }^{1}$ See the recent work [3] for further discussions on the IR universality class of these theories.
} 
is the holomorphic matrix model approach of Dijkgraaf and Vafa [10-12].

The theories of interest to us are obtained from the so-called " $\beta$-deformation" of the $\mathcal{N}=4$ theory with $U(N)$ gauge group. The $\beta$-deformation constitutes one of the two $\mathcal{N}=1$ supersymmetric, exactly marginal deformations of the $\mathcal{N}=4 \mathrm{RG}$ fixed point identified by Leigh and Strassler in [13]. It leads to an $\mathcal{N}=1$ superconformal field theory (SCFT) with three adjoint chiral supermultiplets $X, Y$ and $Z$ which obtain the tree level superpotential interaction ${ }^{2}$

$$
W=\lambda \operatorname{Tr}[X Y Z-q X Z Y] ; \quad q:=e^{i \beta} .
$$

where $\lambda$ and $q$ are complex bare parameters. We also introduce the complex bare gauge coupling of this theory $\tau \equiv 4 \pi i / g_{Y M}^{2}+\theta / 2 \pi$. We will always restrict attention to the case where $q$ is a primitive $n$-th root of unity, $q=\exp (2 \pi i / n)$. In order for the theory to be conformally invariant, the other coupling $\lambda$ has to be some fixed (but unknown) function of $\beta$ and the gauge coupling [13] such that as we approach the $\mathcal{N}=4$ fixed line $(\beta \rightarrow 0)$, we also have $\lambda \rightarrow 1$. The gauge-coupling $\tau$ is a free parameter of the theory and it was shown in [7] that the theory exhibits electricmagnetic duality which acts as $S L(2, \mathbf{Z})$ transformations on the gauge coupling: ${ }^{3}$

$$
\tau \rightarrow \frac{a \tau+b}{c \tau+d}, \quad \beta \rightarrow \frac{\beta}{c \tau+d}
$$

Dynamical phenomena of interest ensue when we break conformal invariance by perturbing the above $\mathcal{N}=1 \mathrm{SCFT}$, with $q=\exp (2 \pi i / n)$, by certain relevant operators. Specifically, we will consider adding to the tree level superpotential, a mass term for one of the fields of the following form

$$
W=\lambda \operatorname{Tr}\left[X Y Z-e^{i \frac{2 \pi}{n}} X Z Y-\epsilon\left(\frac{1}{2} X^{2}-a X\right)\right] ; \quad n>2 .
$$

More complicated polynomial deformations can also be considered leading to similar IR dynamics, but these spoil the UV-behaviour. We also consider slightly more general renormalizable deformation in the penultimate section.

Let us now explain the essential ideas underlying the dynamics of the above theories. It is well known that when $q$ is an $n$-th root of unity the $\beta$-deformation of the $U(N), \mathcal{N}=4$ theory can be realized in IIB string theory as the worldvolume theory of $N$ D3-branes placed at a $\mathbf{C}^{3} / \mathbf{Z}_{n} \times \mathbf{Z}_{n}$ orbifold singularity with a single unit of discrete torsion $[14,15]$. The moduli space of vacua of the $\beta$-deformed theory

\footnotetext{
${ }^{2}$ We work with a normalization where the kinetic terms of the chiral multiplets do not have a factor of $1 / g_{Y M}^{2}$ in front.

${ }^{3}$ Actually, in order to write the transformations in this way the gauge coupling undergoes an algebraic renormalization: we refer to [7] for details.
} 
contains Coulomb and Higgs (and mixed) branches. It follows from the F-term equations that, depending on the rank $N$ of the gauge group, the Higgs branches within the moduli space are symmetric products of a certain number of copies of $\mathbf{C}^{3} / \mathbf{Z}_{n} \times \mathbf{Z}_{n}$. (For example, when $N=m n$ there exists a Higgs branch where the gauge group is broken to a $U(m)$ subgroup and the Higgs branch is a symmetric product of $m$ copies of $\mathbf{C}^{3} / \mathbf{Z}_{n} \times \mathbf{Z}_{n}$ ). The orbifold has three singular (complex) lines which are fixed under the action of the orbifold group, and along which the Coulomb and Higgs branches of the D-brane gauge theory meet. The separations of the D-branes along the fixed lines are the Coulomb branch moduli. At these singular fixed lines there are fractional D-branes bound to the singularities ${ }^{4}$, which can then annihilate in groups of $n$ to form a whole D-brane free to move off into the orbifold bulk (the Higgs branch.)

Once the theory has been deformed by relevant operators, the fixed line singularities above can be smoothed out by complex structure deformations. From the point of view of the gauge theory on the D-branes this corresponds to deforming the superpotential. Deformations that resolve the fixed lines leaving behind only isolated conifold singularities were first discussed in [15]. The deformation (1.3) that we consider was discussed in [6] and [16,17]. After a suitable field redefinition it is equivalent to introducing masses for all three multiplets, a deformation that has been studied for general complex $\beta$ in [7]. Importantly, for the theories with $\beta=2 \pi / n$, this class of deformations does not lift the Higgs branch moduli space, rather it leads to a moduli space which is a complex deformation of the $\mathbf{C}^{3} / \mathbf{Z}_{n} \times \mathbf{Z}_{n}$ orbifold. Classically, the effect of the deformation is to smooth out two of the three fixed lines into a single (complex) hyperboloid, while the third fixed line is completely resolved leaving behind $[n / 2]$ isolated conifold singularities. ${ }^{5}$ ¿From the string theory point of view, once we have isolated conifold singularities in the spacetime geometry we can imagine having fractional branes stuck at these points. Indeed, in the field theory perspective, a bulk D-brane is characterized by an $n$-dimensional irreducible representation of the algebra following from the F-term conditions implied by (1.3). These representations become reducible precisely at each of the $[n / 2]$ points in moduli space corresponding to the conifold singularities. At any one of the conifold singularities labelled by an integer $p$, a bulk D-brane splits up into two lower dimensional irreducible representations of dimension $p$ and $n-p$ respectively. These are the two types of fractional D-branes bound to the singularity.

The appearance of isolated conifold singularities and fractional branes pinned at them naturally points towards the possibility of realizing $\mathcal{N}=1$ gauge theory dynamics of the kind studied by Klebanov and Strassler [2]. This was already realized

\footnotetext{
${ }^{4}$ See [15] for a discussion on the interpretation of these fractional branes.

${ }^{5}$ Note that $[x]$ is the largest integer less than or equal to $x$.
} 
by Berenstein in [4] and [6]. In this paper we will be able to make this precise. First we will show that for the $U(N)$ gauge theory with classical superpotential (1.3), the low energy effective theory at the isolated singular points is a $U\left(N^{+}\right) \times U\left(N^{-}\right)$gauge theory (here $N=p N^{+}+(n-p) N^{-}$) with bi-fundamental matter and a quartic superpotential. In the D-brane language $N^{ \pm}$is the number of fractional branes of each type. In an appropriate regime of parameter space which results in a separation of scales, this theory exhibits a duality cascade that terminates in an $\mathcal{N}=1$ gauge theory with a mass gap. It is important to note that the low-energy theory is not pure $\mathcal{N}=1$ SUSY Yang-Mills theory. In fact it has additional massive fields and is an $\mathcal{N}=1^{*}$ type theory which was studied in [7]. In particular the low energy theory develops a superpotential and gaugino condensate both of which are nontrivial modular functions of the microscopic gauge coupling $\tau$, the same functions that were encountered in [7]. Only in a specific decoupling limit do we obtain pure $\mathcal{N}=1$ SUSY Yang-Mills. All this is encoded rather beautifully in the geometry of the quantum deformed moduli space which we are able to obtain exactly.

The classical moduli space has conifold singularities where fractional branes are pinned. From general considerations $[2,18,19]$ in the quantum theory we expect the fractional branes to deform the geometry further. At the endpoint of the cascade we expect that the (fractional) D-branes have disappeared, the conifold singularities have been replaced by $S^{3}$ 's of finite size resulting in a quantum deformed moduli space seen by a probe D-branes at the endpoint of the cascade. Since all the holomorphic data of an $\mathcal{N}=1$ gauge theory is encoded in a corresponding matrix model [10-12], we should be able to determine this quantum deformed moduli space using the matrix model approach. We achieve this using the fact that the planar sector of the matrix model for our theory is in fact exactly solvable [7,20-22]. Remarkably, in the matrix model one can understand the duality cascade of the field theory in rather simple terms. It turns out that the matrix model description of the field theory vacuum of interest with $U\left(N^{+}\right) \times U\left(N^{-}\right)$gauge symmetry, only depends on a single parameter corresponding to the glueball superfield of the $U\left(\left|N^{+}-N^{-}\right|\right)$gauge group factor appearing at the end of the cascade. Here $\left|N^{+}-N^{-}\right|$has the interpretation as the total number of fractional branes pinned at the conifold singularity. This particular feature was also encountered in [23] in the context of the matrix model description of $\hat{A}_{1}$ quiver theory of [2].

For us the deformed Calabi-Yau geometry emerges as a fibration over a Riemann surface that is completely determined by the matrix model. This quantum geometry is a deformation of the original classical Calabi-Yau space with conifold singularities, and we find that the quantum deformation parameters can be determined exactly as a function of the microscopic gauge coupling $\tau$. The conifold singularities are now replaced by three-cycles whose sizes are exactly calculable, nontrivial functions of 
the gauge coupling or equivalently the glueball superfield. An important feature of these deformed geometries is that each of the "blown-up" $S^{3}$ 's also has a finite dual three-cycle. It is precisely in the limit that this dual cycle is sent to infinity that we are left with just the usual deformed conifold geometry which is dual to pure $\mathcal{N}=1$ SUSY Yang-Mills theory

The layout of this paper is as follows. In Section 2, we review the classical vacuum structure of the field theory and its classical moduli space which has isolated singularities. We describe the classical solutions that correspond to different types of fractional branes stuck at singularities. In Section 3, we obtain the classical effective action for the light modes at the singular points in moduli space and observe that it corresponds (at weak coupling) to the starting point of a duality cascade. In Section 4, by solving the Dijkgraaf-Vafa matrix model associated to this theory we find the quantum deformed moduli space where the deformation parameters are determined precisely in terms of parameters of the gauge theory. Section 5, considers theories with a slightly more general renormalizable deformation including the terms $\operatorname{Tr} X Y$ and $\operatorname{Tr} X Z$. These theories can easily be solved along the lines we set out in earlier sections. Finally, in Section 6 we conclude with some remarks on directions requiring further study. In Appendix A, we derive the quadratic loop equations for the matrix models in question and point out a way to obtain the general loop equations. Various useful results involving elliptic functions are listed in Appendix B.

\section{Classical Vacuum Structure}

In this section we address the question of the classical vacuum structure of the $\beta$ deformed theory with superpotential (1.3). In fact, following [6] we will consider a generalized version of the deformation in (1.3). Since we are primarily interested in the holomorphic structure we shall follow the rule of solving the F-flatness conditions modulo complex gauge transformations. Subject to certain well-known caveats, such solutions are then equivalent to the solution of both the F- and D-flatness conditions up to a complex gauge transformation. We consider the gauge theory perturbed by a polynomial superpotential ${ }^{6}$

$$
W=\operatorname{Tr}[X Y Z-q X Z Y+V(X)], \quad V(x)=\sum_{j \neq 0 \bmod n}^{\ell} \frac{a_{j}}{j} x^{j} .
$$

The fact that we exclude terms in the superpotential proportional to $X^{j}, j=0 \bmod n$ is the restriction of [6] and ensures that the deformation does not lift the Higgs

\footnotetext{
${ }^{6}$ In the rest of the paper we set $\lambda=1$. It could easily be reinstated by by a re-scaling of the fields.
} 
branch. Note that only superpotentials for which $\ell=2$ are actually renormalizable and we shall mostly have this case in mind because we want theories which flow to the superconformal fixed-point in the UV. Note also that in this case once we remove the linear term in $X$ by a shift, the superpotential is equivalent to

$$
W=\operatorname{Tr}\left[X Y Z-q X Z Y-\frac{a_{1}(1-q)}{a_{2}} Y Z+\frac{a_{2}}{2} X^{2}\right],
$$

which with a trivial rescaling is precisely the theory investigated in [7]. However, for illustrative purposes it is also useful to consider the $q=-1$ theory with $\ell=3$ where the superpotential is actually marginally irrelevant, or non-renormalizable.

These perturbed theories and their moduli spaces of vacua have been studied in $[5,6,16,17]$. In what follows, we shall draw heavily on this analysis which shows that the classical moduli space of vacua on a Higgs branch of these theories corresponds to be a 3-complex dimensional Calabi-Yau space $\mathbf{X}$ which is a complex deformation of the orbifold with discrete torsion (i.e. a deformation of $\mathbf{C}^{3} / \mathbf{Z}_{n} \times \mathbf{Z}_{n}$ ). We will further see that the low-energy description of the gauge theory involves both discrete vacua and a moduli space with several branches. On some of these branches, the gauge theory exhibits a subsequent renormalization group cascade of the KlebanovStrassler type [2], and on others the theory flows to the superconformal fixed point of Klebanov and Witten [24]. In addition there are isolated vacua where the theory

confines without undergoing a duality cascade. It is worth mentioning that the existence of this rich vacuum structure is then inherited in the theories related by the $S L(2, \mathbf{Z})$ group. In particular under S-duality, the Higgs branches are mapped to confining branches of the dual theory with modified gauge coupling $1 / \tau$ and a deformation parameter $2 \pi /(n \tau)[7]$.

\subsection{The case $q=-1$ with cubic deformation}

It is instructive to first specialize to the simplest example, namely the theory with $n=2$ (and $q=-1$ ) and subsequently describe the situation for $q$ a generic $n$-th root of unity. Consider then the superconformal theory with $q=-1$ in Eq. (1.1) perturbed by a tree level superpotential for one of the fields $X$. We restrict attention to a perturbation of the form

$$
V(x)=-\epsilon\left(x^{3} / 3-a^{2} x\right)
$$

This perturbation satisfies the restriction implied in Eq. (2.1) which only requires that it should be a polynomial in odd powers of $X$. As we discussed earlier, a cubic superpotential would be marginally irrelevant once quantum effects are taken into account. This fact renders this example less interesting since it spoils the UV 
behaviour. However, it is useful to discuss it because of its simplicity and because it exhibits all the interesting infrared dynamics that we are after. Later we shall consider theories with $n>2$ with quadratic superpotentials which exhibit the same properties but which are also renormalizable. We shall also consider a more general class of theories, including $q=-1$, which are renormalizable.

The F-term equations for the theory with $q=-1$ are

$$
\{X, Y\}=\{X, Z\}=0, \quad\{Y, Z\}=\epsilon\left(X^{2}-a^{2}\right) .
$$

The classical moduli space of this theory can now be obtained following the methods discussed in $[5,6,16]$. First of all, complex gauge transformations can be used to diagonalize, say, $X$. This leaves unfixed diagonal gauge transformations as well as those generated by the Weyl group which act by permutation of the two diagonal elements. We will have more to say about these transformations shortly. For the above choice of potential, the F-term equations are solved by the following set of fields

$$
X=x \sigma^{3} ; \quad Y=y \sigma^{2} ; \quad Z=z \sigma^{1}+z^{\prime} \sigma^{2}
$$

where the $\sigma^{i}$ are Pauli matrices with

$$
2 y z^{\prime}=\epsilon\left(x^{2}-a^{2}\right)
$$

However, this solution is fixed by a $\mathbf{Z}_{2} \times \mathbf{Z}_{2}$ subgroup of the gauge group (the product of the Weyl group and a subgroup of the diagonal transformations). Explicitly the two generators are

$$
\theta_{1}=\sigma_{3}, \quad \theta_{2}=\sigma_{1} .
$$

Modding out by these transformations is equivalent to identifying

$$
\theta_{1}: \quad(x, y, z) \sim(x,-y,-z), \quad \theta_{2}: \quad(x, y, z) \sim(-x,-y, z)
$$

In the D-brane interpretation of $[16,17]$ this irreducible $2 \times 2$ dimensional representation should be thought of as parameterizing the moduli space of a single D3-brane probe moving on a 3-complex dimensional Calabi-Yau space $\mathbf{X}$. In order to find an explicit form for this classical moduli space we need to introduce gauge invariant variables which automatically incorporate the identifications (2.8). This can be done by first enumerating the set of elements that commute with all the generators of the algebra in Eq. (2.4). One finds $X^{2}, Y^{2}$ and $Z^{2}$. These 3 generators are therefore proportional to the identity matrix and we can introduce the gauge invariant variables $(u, v, w)$ via

$$
u \cdot 1_{[2] \times[2]}=X^{2}, \quad v \cdot 1_{[2] \times[2]}=Y^{2}, \quad w \cdot 1_{[2] \times[2]}=Z^{2} .
$$


Clearly

$$
u=x^{2}, \quad v=y^{2}, \quad w=z^{2}+z^{\prime 2} .
$$

There is an additional central element:

$$
t \cdot 1_{[2] \times[2]}=X Y Z+\frac{1}{2} X V^{\prime}(X)
$$

The four variables $(t, u, v, w)$ are not independent, rather they satisfy the relation

$$
t^{2}=-u v w+\frac{\epsilon^{2}}{4} u\left(u-a^{2}\right)^{2}
$$

This algebraic equation describes a (non-compact) 3-complex dimensional CalabiYau space $\mathbf{X}$ in $\mathbf{C}^{4}$ which is precisely a complex deformation of the $\mathbf{C}^{3} / \mathbf{Z}_{2} \times \mathbf{Z}_{2}$, the orbifold with discrete torsion. Indeed, when the deformation vanishes we have $t^{2}=-u v w$ which is the well-known algebraic description of the orbifold. The undeformed orbifold with discrete torsion has (classical) singularities corresponding to the (complex) fixed lines where any two of $u, v$ and $w$ vanish. These fixed lines correspond to solutions (2.5) (with $\epsilon=0$ ) fixed by $\theta_{1}, \theta_{2}$ or $\theta_{1} \theta_{2}$, so $y=z=0$, $x=y=0$ and $x=z=0$, respectively.

The addition of the superpotential perturbation $(\epsilon \neq 0)$ resolves the orbifold singularities, but only partially. In particular, it is easy to see that the singular lines associated to the undeformed solution fixed by the gauge transformations $\theta_{2}$ and $\theta_{1} \theta_{2}(u=v=0$ and $u=w=0$, respectively) merge. For instance, the singular line associated to $\theta_{1} \theta_{2}$ becomes $x=z=0$ and $y z^{\prime}=-\epsilon a^{2} / 2$, or $u=0$ and $w v=\left(-\epsilon a^{2} / 2\right)^{2}$. However, the third singular line associated to the solution fixed by $\theta_{1}$ is reduced to an isolated singular point located at $\left(u=a^{2}, v=0, w=0\right)$. This singularity is locally of the conifold type. ${ }^{7}$ In fact precisely as we approach this singular point from the bulk, the two-dimensional solutions to the F-term equations above become reducible in the sense that there are now two one-dimensional solutions $y=z=0$ and $x= \pm a$. In the brane interpretation, the fact that the two-dimensional irreducible representations of the deformed algebra split up into two one-dimensional representations can be interpreted as the fractionation of a bulk brane at the conifold singularity. In particular each one-dimensional solution corresponds to a fractional brane stuck at the conifold singularity while the irreducible two-dimensional solution describes the possibility for the two types of fractional branes at the singularity to combine into a whole brane and move away from the singular point into the bulk of the deformed geometry which is also the Higgs branch of the D-brane gauge theory. (Note that the one-dimensional solutions only exist at $u=a^{2}$ and so an individual fractional brane can only be pinned at this singularity.) In the superconformal theory

\footnotetext{
${ }^{7}$ This can be seen by introducing the new variable $\delta=u-a^{2}$ and focusing attention on $\delta, v, w \ll$ $a^{2}$.
} 
with $\epsilon=0$ this conifold singularity becomes a point on the fixed line $v=w=0$ where the Coulomb branch meets the Higgs branch.

\subsection{The general case $n>2$}

The picture that emerges for generic $n>2$ is quite similar. If we are only interested in the low-energy dynamics we can always allow arbitrary polynomial perturbations satisfying the requirement (2.1) resulting in classically deformed moduli spaces that will be described below. The restriction to renormalizable terms will become important in the context of what we have to say in the next section.

The F-term equations for the perturbed theory are

$$
X Y-q Y X=0, \quad Z X-q X Z=0, \quad Y Z-q Z Y=-V^{\prime}(X)
$$

To start with there are two Coulomb branches where $X=Z=0$ and $Y$ diagonal and $X=Y=0$ and $Z$ diagonal, respectively. We will not be interested in these branches. The remaining solutions can be built out of irreducible blocks of size up to $n$. Blocks of dimension $p<n$ come in sets $\left\{\mathfrak{R}_{i}^{(p)}\right\}$ while the block $\mathfrak{R}^{(n)}$ of dimension $n$ is unique. In the $q=-1$ case above these were the two one-dimensional solutions and the twodimensional irreducible solution, respectively. The solutions with dimension $p<n$, the $\left\{\mathfrak{R}_{i}^{(p)}\right\}$, will describe fractional branes pinned at conifold singularities. The other irreducible $n$-dimensional solution $\mathfrak{R}^{(n)}$ will describe whole branes that are free to move off the singularities and explore the bulk of the classical moduli space.

In the following a central rôle will be played by the function $f(x)$ defined in terms of the potential by

$$
x V^{\prime}(x)=f(x)-f(x q) .
$$

Note that

$$
f(x)=\sum_{j \neq 0 \bmod n}^{\ell} \frac{a_{j} x^{j}}{1-q^{j}}
$$

and has the same order as $V(x)$. The two classes of solutions to (2.13) are more specifically 
(i) $\mathfrak{R}_{i}^{(p)}, i=1, \ldots, d^{(p)}$, of dimension $p<n$ :

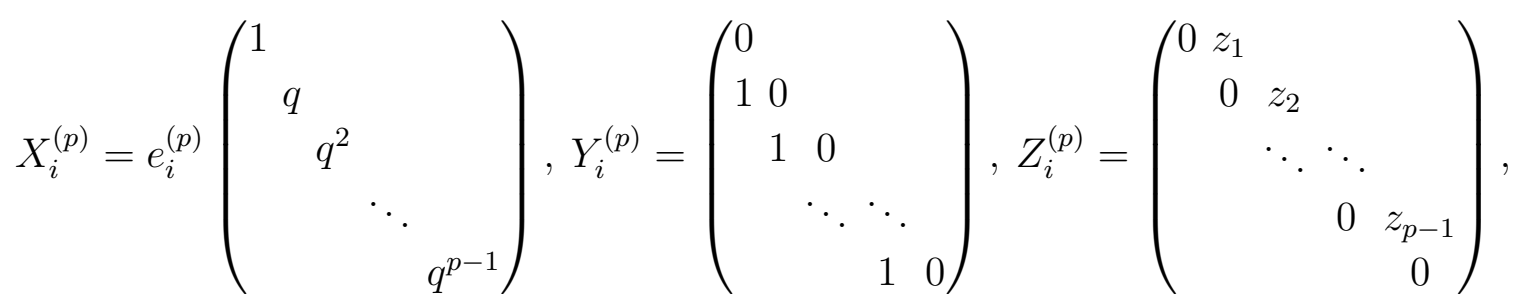

where $e_{i}^{(p)}$ is a root of the polynomial equation

$$
f(x)=f\left(x q^{p}\right)
$$

excluding $x=0$.

(ii) $\mathfrak{R}^{(n)}\left[u=x^{n}, v=y^{n}, w=z^{n}\right]$, of dimension $n$ :

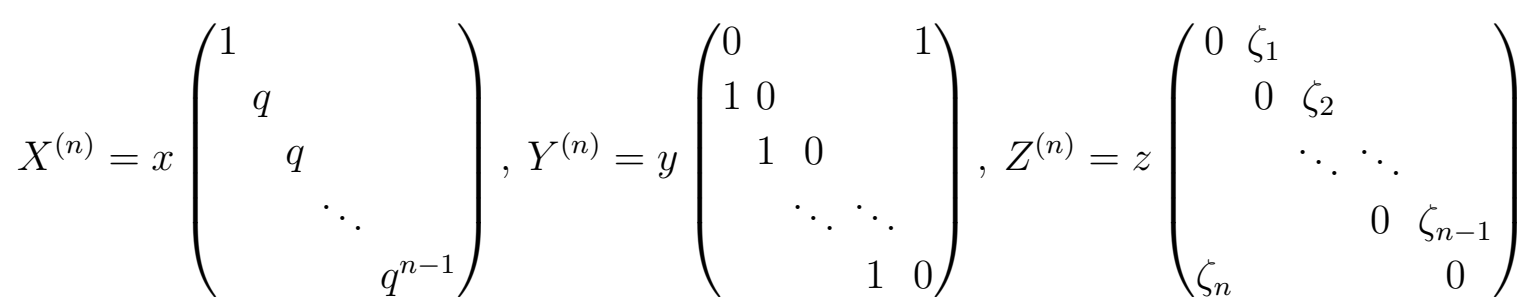

with $\prod_{j=1}^{n} \zeta_{j}=1$. The actual values of the $\zeta_{i}$ are fixed but will not be required.

Since $x, y$ and $z$ are not fixed the representation $\mathfrak{R}^{(n)}[u, v, w]$ has a moduli space which can be obtained, as in the $q=-1$ case, by determining the relation between the elements of the center of the algebra, namely

$u \cdot 1_{[n] \times[n]}=X^{n}, \quad v \cdot 1_{[n] \times[n]}=Y^{n}, \quad w \cdot 1_{[n] \times[n]}=Z^{n}, \quad t \cdot 1_{[n] \times[n]}=X Y Z+f(X)$.

The set of variables $(t, u, v, w)$ satisfy the relation

$$
u v w=(-1)^{n+1} \prod_{j=1}^{n}\left(t-f\left(x q^{j}\right)\right)=(-1)^{n+1} F(t, u) .
$$

Notice that the right hand side of this equation is actually invariant under $x \rightarrow q x$ and can therefore be only a polynomial $F(t, u)$ in the invariant $u=x^{n}$. This is a 3-complex dimensional Calabi-Yau space $\mathbf{X}$ which is a deformation of the orbifold $\mathbf{C}^{3} / \mathbf{Z}_{n} \times \mathbf{Z}_{n}$ with discrete torsion described by the algebraic equation $t^{n}=$ $(-1)^{n+1} u v w$.

As in the $q=-1$ case the singular fixed lines of the orbifold are partially resolved. Rather than investigating these singularities by considering the action of 
gauge transformation as we did for $q=-1$, the structure of the singularities can equivalently be determined directly from the Calabi-Yau geometry (2.20), as in [6], by demanding that the partial derivatives with respect to $u, v, w$ and $t$ vanish. The first kind correspond to $u=0$ along with

$$
v w=\left.\partial_{u} F(t, u)\right|_{u=0},
$$

which is a deformation of the two fixed lines $u=v=0$ and $u=w=0$. The other class of singularities are the ones that interest us. They are isolated points in the geometry where $v=w=0$ and the the $n$-sheeted algebraic curve, or Riemann surface, $\Sigma$ defined by

$$
F(t, u)=0
$$

becomes singular. These points are simply given by the roots of

$$
f(x)=f\left(x q^{p}\right)
$$

for any $p<n$, excluding $x=0$. Hence the isolated singular points are precisely at the locations of the fractional branes associated to the representation $\mathfrak{R}_{i}^{(p)}$. It

Earlier we denoted these roots $e_{i}^{(p)}$, where $i$ counts the degeneracy: $i=1, \ldots, d^{(p)}$. The fractional branes are pinned at a conifold-type singularity at

$$
u=u_{i}^{(p)} \equiv\left(e_{i}^{(p)}\right)^{n}, \quad v=w=0 .
$$

Let us enumerate the number of these isolated singular points. Recall that we are counting the roots of $f(x)-f\left(x q^{p}\right)$ which is a polynomial equation of degree $\ell$, except when $\ell p=0 \bmod n$, in which case it has degree $\ell-1$. So the number of roots, apart from $x=0$, is

$$
d^{(p)}= \begin{cases}\ell-2 & \ell p=0 \bmod n \\ \ell-1 & \text { otherwise }\end{cases}
$$

Note also that there is a symmetry

$$
e_{i}^{(n-p)}=q^{p} e_{d^{(p)}+1-i}^{(p)}
$$

and $d^{(p)}=d^{(n-p)}$. (These equivalences are valid when $p=n / 2$ for $n$ even.) This means that the fractional branes associated to $\mathfrak{R}_{i}^{(p)}$ and $\mathfrak{R}_{d^{(p)}+1-i}^{(n-p)}$ lie at the same point in the geometry:

$$
u_{i}^{(p)}=u_{d(p)+1-i}^{(n-p)} .
$$

This is very familiar from the brane interpretation of the basic conifold theory: fractional branes come in pairs. In order to avoid this cumbersome notation, we shall label the singularities, or pairs of mirror fractional branes, by the index $\mu=(p, i)$. 
In particular, for a given $\mu$ the associated value of $p$ is $1 \leq p_{\mu} \leq[n / 2]$ and we denote the mirror pair $\mathfrak{R}_{i}^{(p)}$ and $\mathfrak{R}_{d^{(p)}+1-i}^{(n-p)}$ as $\mathfrak{R}_{\mu}^{+}$and $\mathfrak{R}_{\mu}^{-}$. Finally

$$
u_{\mu} \equiv u_{i}^{(p)}=u_{d^{(p)}+1-i}^{(n-p)}, \quad \mu=1, \ldots, g
$$

Note that the number of singularities, or pairs of fractional branes, is equal to

$$
g=\frac{d^{(n / 2)}}{2}+\sum_{j=1}^{[(n-1) / 2]} d^{(p)}
$$

However, can we see that if we have two fractional branes, one of each type, then it can become a "complete" brane and move off into the bulk? The answer is yes because at $\left(u=u_{\mu}, v=0, w=0\right)$ the representation $\mathfrak{R}^{(n)}[u, v, w]$ is actually reducible:

$$
\mathfrak{R}^{(n)}\left[u=u_{\mu}, v=0, w=0\right] \simeq \mathfrak{R}_{\mu}^{+} \oplus \mathfrak{R}_{\mu}^{-}
$$

Let us consider in more detail the relation of these singularities to the algebraic curve $\Sigma$ defined by $F(t, u)=0$. We can think of $\Sigma$ as an $n$-fold cover the $u$ plane. There is a $n^{\text {th }}$-order branch cut running from $u=0$ to $u=\infty$ which joins all the sheets. In addition for $u=u_{\mu}$ a pair of sheets touch at a point. So as one expects the curve $\Sigma$ is singular at these points. In the quantum theory the picture emerging will be that the presence of a net number fractional branes, i.e. the number of representations $\mathfrak{R}_{\mu}^{+}$minus the number of $\mathfrak{R}_{\mu}^{-}$, has the effect of deforming the isolated conifold singularity in $\mathbf{X}$ which at the level of the curve $\Sigma$ involves the opening up of the point $u=u_{\mu}$ into a cut joining the pair of sheets.

Now that we have described the irreducible blocks, we can now write down a general classical vacuum. If one starts with a $U(N)$ gauge group then a general vacuum is associated to a reducible representation

$$
\oplus_{\mu=1}^{g}\left(N_{\mu}^{+} \cdot \mathfrak{R}_{\mu} \oplus N_{\mu}^{-} \cdot \mathfrak{R}_{\mu}^{-}\right)
$$

where

$$
N=\sum_{\mu=1}^{g}\left(p_{\mu} N_{\mu}^{+}+\left(n-p_{\mu}\right) N_{\mu}^{-}\right) .
$$

Notice that we didn't include the $\mathfrak{R}^{(n)}$ block explicitly because the vacuum (2.31) lies at a point in a large moduli space where up to $\left|N_{\mu}^{+}-N_{\mu}^{-}\right|$, for each pair $\mathfrak{R}_{\mu}^{ \pm}$of fractional branes, can move of into the bulk and explore the space $\mathbf{X}$. 


\section{The Classical Effective Action and RG Cascade}

We have seen that certain deformations of the $\beta$-deformed $\mathcal{N}=4$ theory lead to deformed (classical) moduli spaces for D-branes with isolated conifold singularities where fractional branes can be stuck. We will now ask what the effective gauge theory is on stacks of branes some of which remain stuck at the isolated points while others have moved off to explore the full moduli space. As in the last Section it is useful to consider the $q=-1$ case first.

\subsection{The case $q=-1$ with cubic deformation}

Consider the $U(N)$ gauge theory with the cubic plus linear superpotential (2.3). Recall that there is a single isolated singularity in $\mathbf{X}$ at $u=a^{2}, v=w=0$. The two fractional branes are distinguished by $x= \pm a$. So let us consider a vacuum with $N^{+}$ (fractional) branes with $x=+a$ and $N^{-}$branes with $x=-a$ (fractional branes of the other type) with $N^{+}+N^{-}=N$. In this vacuum

$$
X=\left(\begin{array}{cc}
a \cdot 1_{\left[N^{+}\right] \times\left[N^{+}\right]} & 0 \\
0 & -a \cdot 1_{\left[N^{-}\right] \times\left[N^{-}\right]}
\end{array}\right), \quad Y=Z=0 .
$$

Note that as we remove the deformation smoothly $V \rightarrow 0$, these points lie at the intersection of the Coulomb and Higgs branches of the parent superconformal theory. The classical vacuum (3.1) preserves a $U\left(N^{+}\right) \times U\left(N^{-}\right)$gauge symmetry.

The low-energy classical $U\left(N^{+}\right) \times U\left(N^{-}\right)$gauge theory at these points in moduli space is obtained by expanding in fluctuations about the above solution,

$$
\delta X=\left(\begin{array}{cc}
X_{1} & 0 \\
0 & X_{2}
\end{array}\right), \quad \delta Y=\left(\begin{array}{cc}
Y_{1} & A_{1} \\
B_{1} & Y_{2}
\end{array}\right), \quad \delta Z=\left(\begin{array}{cc}
Z_{1} & A_{2} \\
B_{2} & Z_{2}
\end{array}\right)
$$

where $X_{1}, Y_{1}$ and $Z_{1}$ transform in the adjoint representation of the unbroken $U\left(N^{+}\right)$, while $X_{2}, Y_{2}$ and $Z_{2}$ are in the adjoint of the $U\left(N^{-}\right)$. In addition the fields $A_{i}$ and $B_{i}$ fill out four bi-fundamental multiplets transforming as $\left(N^{+}, \bar{N}^{-}\right)$and $\left(\bar{N}^{+}, N^{-}\right)$ respectively.

The nature of the low-energy theory can now be easily determined by expanding out the classical superpotential in terms of the fluctuations and we find

$$
\begin{aligned}
W & =\operatorname{Tr}\left[X_{1}\left(A_{1} B_{2}+A_{2} B_{1}\right)-\epsilon a X_{1}^{2}-\frac{\epsilon}{3} X_{1}^{3}+2 a Y_{1} Z_{1}+X_{1}\left(Z_{1} Y_{1}+Y_{1} Z_{1}\right)\right] \\
& +\operatorname{Tr}\left[X_{2}\left(B_{1} A_{2}+B_{2} A_{1}\right)+\epsilon a X_{2}^{2}-\frac{\epsilon}{3} X_{2}^{3}-2 a Y_{2} Z_{2}+X_{2}\left(Y_{2} Z_{2}+Z_{2} Y_{2}\right)\right]
\end{aligned}
$$


Note that the fluctuations $Y_{1,2}$ and $Z_{1,2}$ have a mass set by the scale $a$ while those associated to $X_{1}$ and $X_{2}$ have a mass set by the scale $\epsilon a$. Assuming $\epsilon \ll 1$ and the gauge coupling is weak enough, it makes sense we can integrate out $Y_{1,2}$ and $Z_{1,2}$ at the classical level to arrive at the effective superpotential

$$
W_{\text {eff }}=\operatorname{Tr}\left[X_{1}\left(A_{1} B_{2}+A_{2} B_{1}\right)-\epsilon a X_{1}^{2}\right]+\operatorname{Tr}\left[X_{2}\left(B_{1} A_{2}+B_{2} A_{1}\right)+\epsilon a X_{2}^{2}\right]
$$

where we have neglected the cubic coupling of $X_{1,2}$ which will be irrelevant in the IR. The effective theory for the light modes is a mass deformation of the $U\left(N^{+}\right) \times U\left(N^{-}\right)$ $\mathcal{N}=2$ theory with equal and opposite sign masses for the two adjoint scalars. This is precisely the starting point of the Klebanov-Strassler duality cascade. We can also now integrate out $X_{1,2}$ to arrive at

$$
W_{\text {eff }}=-\frac{1}{4 \epsilon a} \operatorname{Tr}\left(A_{i} B_{j} A_{k} B_{l}\right) \epsilon^{i k} \epsilon^{j l} .
$$

For a fixed given value of $N$ we can vary $N^{+}\left(\right.$or $\left.N^{-}\right)$and explore different vacua of the theory. In particular when the unbroken rank $N$ is even we can choose the vacuum with $N^{+}=N^{-}=N / 2$. Here the resulting $U(N / 2) \times U(N / 2)$ theory with bi-fundamentals and the quartic superpotential above flows to the superconformal conifold theory of Klebanov and Witten [24]. As pointed out in [24], the quartic superpotential is actually an exactly marginal operator at the IR fixed point. Thus we have found a flow that interpolates between the $\mathbf{C}^{3} / \mathbf{Z}_{2} \times \mathbf{Z}_{2}$ theory and the Klebanov-Witten conifold theory.

For any given $N=N^{+}+N^{-}$we can sweep through distinct classical vacua by varying $N^{+}$. In particular, when $N^{+}=0$, we simply have a $U(N)$ gauge theory with $\mathcal{N}=1$ SUSY with massive adjoint scalars. The $S U(N)$ subgroup of the $U(N)$ gauge group confines and generates a mass gap and in many ways is similar to the confining vacuum of $\mathcal{N}=1^{*}$ gauge theory $[8,27]$. In an appropriate decoupling limit for the adjoint scalars, the theory reduces to pure $\mathcal{N}=1$ SUSY gauge theory.

The remaining vacua labelled by generic values of $N^{+}$contain rich and complicated dynamics. In particular, at least at weak coupling $g_{Y M}^{2} \ll 1$ (the gaugecoupling of the parent $q=-1$ SCFT), the low-energy $U\left(N^{+}\right) \times U\left(N^{-}\right)$theory with bi-fundamentals and a quartic superpotential shares all the features of the theory studied by Klebanov and Strassler [2]. Under renormalization group flow, the individual gauge group factors will undergo successive Seiberg dualities that terminate at an $\mathcal{N}=1$ theory with a mass gap and gaugino condensation, with or without probe branes propagating on a deformed and desingularized moduli space. We will return to a more detailed quantum description of these vacua after demonstrating how they arise in the theories with $n>2$. 


\subsection{The case $n>2$ with quadratic deformation}

At the outset we remark that there is a key difference between the cascade theories encountered here (and above) and that of Klebanov-Strassler. The theory considered in [2] has the property that one of the gauge group factors has a Landau pole in the ultraviolet. (One could imagine achieving a UV completion by a reverse duality cascade, but in that case the ranks of the gauge group factors would grow without bound in the $\mathrm{UV}^{8}$.) The theories we consider have the property that they are obtained as deformations of a four dimensional $\mathcal{N}=1$ superconformal field theory with $U(N)$ gauge group and therefore any potential duality cascade is embedded within a well-defined UV-theory. In this context, note that although the deforming potential for $q=-1$ was classically marginal (i.e. cubic), it is most likely to be rendered irrelevant by quantum effects. However, as we will see below for the theories with $n>2$ the deforming potentials need only be quadratic-hence renormalizable - to obtain an RG cascade in the infrared.

We consider the superconformal theory perturbed by the superpotential

$$
V(x)=-\epsilon\left(x^{2} / 2-a x\right)
$$

This superpotential yields exactly one fractional brane solution for each irreducible representation of dimension $p<n$ (except for $p=n / 2$ when $n$ is even) stuck at

$$
u=u^{(p)}=\left(a \frac{1+q}{1+q^{p}}\right)^{n}, \quad v=w=0
$$

in $\mathbf{X}$. We now consider the theory with a $U(N), N=p N^{+}+(n-p) N^{-}$gauge group in a vacuum with $N^{+}$fractional $\mathfrak{R}^{(p)}$ branes and $N^{-}$fractional $\mathfrak{R}^{(n-p)}$ branes. We are going to argue that as in the $q=-1$ case the classical effective theory is a $U\left(N^{+}\right) \times U\left(N^{-}\right)$theory with massless bi-fundamentals. First-of-all, the gauge group is obviously broken to $U\left(N^{+}\right) \times U\left(N^{-}\right)$. This is clear from the from of the VEV assigned to the adjoint field $X$ :

$$
X=\left(\begin{array}{cc}
1_{\left[N^{+}\right] \times\left[N^{+}\right]} \otimes X^{(p)} & 0 \\
0 & 1_{\left[N^{-}\right] \times\left[N^{-}\right]} \otimes X^{(n-p)}
\end{array}\right) .
$$

But the question is whether there are the right number of massless bi-fundamental fields. Indeed the answer is yes, as we can see by first considering the simpler case of the theory realized on one $\mathfrak{R}^{(p)}$ brane and one $\mathfrak{R}^{(n-p)}$ brane. As we have already seen, these two fractional branes make a complete brane that can move off onto the Higgs branch and thus there must be additional massless moduli. These additional moduli

\footnotetext{
${ }^{8}$ For a discussion of the UV behaviour of various theories exhibiting the phenomenon of duality cascades, see $[25,26]$
} 
correspond to the non-zero elements of $Y$ and $Z$ in the representation $\mathfrak{R}^{(n)}[u, v, w]$ that are zero in $\mathfrak{R}^{(p)} \oplus \mathfrak{R}^{(n-p)}$. More specifically if $E_{i, j}$ is a matrix with a 1 in position $(i j)$ and zeros elsewhere, then the elements in question are $E_{p+1, p}$ and $E_{1, n}$ in $Y$ and $E_{p, p+1}$ and $E_{n, 1}$ in $Z$.

Therefore, when we have $N^{+}$coincident $\mathfrak{R}^{(p)}$ branes and $N^{-} \mathfrak{R}^{(n-p)}$ branes at the singular point there will be $4 \times N^{+} \times N^{-}$such massless fields which will fill out a bi-fundamental multiplet in the $\left(N^{+}, \bar{N}^{-}\right) \oplus\left(\bar{N}^{+}, N^{-}\right)$of the low-energy product gauge group. To be more specific the low-energy degrees of freedom can be encoded in the following set of fluctuating modes

$$
\begin{aligned}
\delta X & =\left(\begin{array}{cc}
X_{1} \otimes P^{(p)} & 0 \\
0 & X_{2} \otimes q^{p} P^{(n-p)}
\end{array}\right)+\tilde{\delta} X, \\
\delta Y & =\left(\begin{array}{cc}
0 & A_{1} \otimes E_{1, n-p} \\
B_{1} \otimes E_{1, p} & 0
\end{array}\right)+\tilde{\delta} Y, \\
\delta Z & =\left(\begin{array}{cc}
0 & q^{-p} A_{2} \otimes E_{p, 1} \\
B_{2} \otimes E_{n-p, 1} & 0
\end{array}\right)+\tilde{\delta} Z .
\end{aligned}
$$

Here $X_{1}$ transforms in the adjoint representation of the unbroken $U\left(N^{+}\right)$while $X_{2}$ transforms in the adjoint of $U\left(N^{-}\right)$. We have also defined $P^{(p)}=\operatorname{diag}\left(1, q, \ldots, q^{p-1}\right)$. The modes $\left(A_{i}, B_{i}\right)$ are the bi-fundamentals transforming in the $\left(N^{+}, \bar{N}^{-}\right)$and $\left(\bar{N}^{+}, N^{-}\right)$representations, respectively. The remaining fluctuations (modulo gauge transformations) are denoted $\tilde{\delta} X$, etc.. The classical superpotential for the fluctuations at the chosen vacuum is then

$$
\begin{gathered}
\frac{1}{W}=\operatorname{Tr}\left(X_{1}\left(A_{1} B_{2}-A_{2} B_{1}\right)\right)+\operatorname{Tr}\left(X_{2}\left(B_{1} A_{2}-B_{2} A_{1}\right)\right) \\
+\frac{\epsilon}{2} \cdot \frac{1-q^{2 p}}{1-q^{2}}\left(\operatorname{Tr} X_{1}^{2}-\operatorname{Tr} X_{2}^{2}\right)+\tilde{\delta} W .
\end{gathered}
$$

The remaining factor $\tilde{\delta} W$ includes all the additional fluctuating modes. Importantly all these modes gain a mass at a scale $a$ and so for sufficiently large $a$ compared with $\epsilon$ there is a separation-of-scales between these tilded modes and the ones we have singled out and we can ignore them - at least at sufficiently weak coupling. Note that the effective couplings of the $U\left(N^{+}\right)$and $U\left(N^{-}\right)$factors are $p \tau$ and $(n-p) \tau$, respectively.

We might have expected the above picture to be true since from a geometric point-of-view taking $a \gg \epsilon$ zeros-in on the singularity which is then well approximated by the deformed conifold and the usual story of the duality cascade and deformed conifold should then be applicable [2]. Indeed upon integrating out the massive adjoints we obtain precisely, the quartic superpotential

$$
W_{\text {eff }}=-\frac{1}{2 \epsilon} \cdot \frac{1-q^{2}}{1-q^{2 p}} \operatorname{Tr}\left(A_{i} B_{j} A_{k} B_{l}\right) \epsilon^{i k} \epsilon^{j l} .
$$


Note that we have obtained what will be the starting point for a duality cascade under renormalization group flow, specifically when the gauge coupling of the ultraviolet theory is taken to be sufficiently weak in order that the effective superpotential (3.10) is valid over a certain range of energies between the decoupling of the additional modes and the onset of the cascade. What is new and remarkable is that the duality cascade has been embedded in a four-dimensional theory with a perfectly well-defined UV limit given by the superconformal fixed point. The RG flow away from the fixed point is defined by the mass deformation (3.6). The end-point of the cascade is a $U\left(\left|N^{+}-N^{-}\right|\right)$theory which confines. As noted earlier, when $N^{+}=N^{-}$the theory flows to the conifold superconformal theory realizing a flow between the $\mathbf{C}^{3} / \mathbf{Z}_{n} \times \mathbf{Z}_{n}$ orbifold and the Klebanov-Witten SCFT.

It is clearly possible to generalize the discussion to a vacuum with more than one type of fractional brane pair. In this case, at low energy the theories associated to each pair will decouple from each other.

\section{Engineering the Quantum Geometry from the Matrix Model}

We have seen how the classical low-energy description of the perturbed SCFT leads to a picture of the gauge theory being realized on (fractional) D-branes placed at conifold singularities in a geometry $\mathbf{X}$ which is a deformation of the $\mathbf{C}^{3} / \mathbf{Z}_{n} \times \mathbf{Z}_{n}$ orbifold. This deformed geometry was precisely the Higgs branch moduli space of the gauge theory. ¿From the classical picture we also concluded that at generic classical vacua the theory undergoes strong-coupling quantum dynamics such as successive Seiberg dualities leading to a duality cascade and/or confinement with gaugino condensation. We expect that the nonperturbative quantum effects (i.e. the presence of fractional branes) should result in a further deformation of the geometry above which desingularizes the isolated conifold points. This was exactly the picture at the endpoint of the RG cascade in [2] wherein the associated geometry was just the deformed conifold. But this cannot be the whole story in the class of theories that we are studying as they are actually UV finite theories that confine in the IR with or without an intermediate duality cascade. In a certain decoupling limit for the adjoint scalars they will reduce precisely to the theory of [2]. Correspondingly, the deformed Calabi-Yau geometry $\mathbf{X}$ (which will be the moduli space seen by probe branes at the end of a cascade) will only reduce to the deformed conifold in a limit where some 3 -cycle is sent to infinity.

¿From the work of Dijkgraaf and Vafa [10-12], it is now well-understood how to obtain the geometry associated to a given $\mathcal{N}=1$ gauge theory. In particular, one 
engineers the gauge theory on D-branes in a Calabi-Yau geometry. Via a geometric transition the system has a dual description in terms of a deformed Calabi-Yau geometry with fluxes and no D-branes and this geometry is captured by a certain Riemann surface encoded in a specific matrix model.

The matrix model associated to our theory is defined by the partition function

$$
\mathcal{Z}=\frac{1}{\operatorname{Vol}[U(\hat{N})]} \int[d X]_{\hat{N}^{2}}[d Y]_{\hat{N}^{2}}[d Z]_{\hat{N}^{2}} \exp \left[-\frac{1}{g_{s}} \operatorname{Tr}(X Y Z-q X Z Y+V(X))\right] .
$$

where there is matrix for each chiral field of the theory. As usual the dimensions of the matrices indicated by $\hat{N}$ are not the same as the fields in the parent field theory.

As in earlier works $[7,20,21]$ and references therein, the holomorphic integral is to be thought of as a contour integral and in practice is performed by choosing $Y=Z^{\dagger}$ and then integrating them out. The resulting one-matrix model can be re-written as an ordinary $\hat{N}$-dimensional integral in terms of the eigenvalues $\left\{x_{i}\right\}$ of $X$ :

$$
\mathcal{Z}=\int \prod_{i=1}^{\hat{N}} d x_{i} \frac{\prod_{i \neq j} x_{i}-x_{j}}{\prod_{i, j} x_{i}-q x_{j}} e^{-\frac{1}{g_{s}} \sum_{i} V\left(x_{i}\right)} .
$$

\subsection{Saddle point condition}

The connection with the holomorphic sector of the gauge theory and the associated Riemann surface emerges from the so-called genus zero contributions to the matrix integral when evaluated in a 't Hooft large- $\hat{N}$ expansion around a saddle-point. In this limit, $\hat{N} \rightarrow \infty$ and $g_{s} \rightarrow 0$ with $S=g_{s} \hat{N}$ fixed. The saddle point configuration of the eigenvalues $\left\{x_{i}\right\}$ is determined by extremizing the effective action (4.2) leading to the condition

$$
g_{s}\left[2 \sum_{j \neq i} \frac{1}{x_{i}-x_{j}}-\sum_{j} \frac{1}{x_{i}-q x_{j}}-\sum_{j} \frac{1}{x_{i}-q^{-1} x_{j}}\right]=V^{\prime}\left(x_{i}\right) .
$$

In practice, one starts with a classical saddle point solution (of the field theory), where the left-hand side of (4.3) vanishes, described by a set of eigenvalues $\left\{x_{a}\right\}$ with a certain degeneracy $N_{a}, \sum_{a} N_{a}=N$. In the matrix model, one replaces $N_{a} \rightarrow \hat{N}_{a}$, $\sum_{a} \hat{N}_{a}=\hat{N}$, and takes $\hat{N}_{a} \rightarrow \infty$ and $g_{s} \rightarrow 0$ with each $\hat{S}_{a}=g_{s} \hat{N}_{a}$ fixed. In this limit, the basic assumption is that the eigenvalues at $x_{a}$ spread out in a one-dimensional distribution on an open contour $\mathcal{C}_{a}$ in the complex $x$-plane. This distribution can be described by a unit-normalized density $\rho(x)$ with support on the union of contours $\mathcal{C}=\cup_{a} \mathcal{C}_{a}$. The density of eigenvalues can be encoded in the resolvent function

$$
\omega(x)=\lim _{\hat{N} \rightarrow \infty} \frac{1}{\hat{N}} \sum_{i} \frac{1}{x-x_{i}}=\int_{\mathcal{C}} d y \frac{\rho(y)}{x-y} .
$$


The resolvent $\omega(x)$ is analytic and only has cuts along the equilibrium distribution with a discontinuity that determines the density:

$$
\omega(x+\epsilon)-\omega(x-\epsilon)=2 \pi i \rho(x), \quad x \in \mathcal{C},
$$

where $\epsilon$ is a suitable infinitesimal. Integrating the resolvent over any one of its branch cuts $\mathcal{C}_{a}$ gives us the fraction of the total number of eigenvalues, $\hat{N}_{a} / \hat{N}$, that are distributed along that cut.

The saddle-point equation (4.3) in the large $\hat{N}$ limit can now be written in terms of the resolvent function as

$$
S\left[\omega(x+\epsilon)+\omega(x-\epsilon)-q \omega(q x)-q^{-1} \omega\left(q^{-1} x\right)\right]=V^{\prime}(x), \quad x \in \mathcal{C},
$$

where $\epsilon$ is a suitable infinitesimal so that $x \pm \epsilon$ lie above and below the cut at $x \in \mathcal{C}$. The content of this saddle point equation is easily understood when recast in terms of a new function $t(x)$ (see also [7,20-22]) which naturally encodes the geometry of the quantum deformed moduli space and will also allow us to see the duality cascade of the field theory in a simple way. The form of the saddle point equation (4.6) motivates us to define

$$
t(x):=f(x)+S x\left(q^{-1} \omega\left(q^{-1} x\right)-\omega(x)\right)
$$

where $f(x)$ was introduced in (2.14). From the analytic structure of the resolvent $\omega(x)$ it follows that $t(x)$ has cuts at $\mathcal{C}=\cup_{a} \mathcal{C}_{a}$ and its rotation by $q: q \mathcal{C}=\cup_{a}\left(q \mathcal{C}_{a}\right)$. The saddle-point equation (4.6) is then very simple:

$$
t(x \pm \epsilon)=t(q(x \mp \epsilon)), \quad x \in \mathcal{C} .
$$

This is simply a gluing condition that glues a point $x \pm \epsilon$ on the top/bottom of the cut $\mathcal{C}_{a}$ to a point $q(x \mp \epsilon)$ on the bottom/top of the cut $q \mathcal{C}_{a}$. So $t$ defines a Riemann surface $\tilde{\Sigma}$ which is a copy of the complex $x$-plane with the cuts identified as above.

Before we describe the solution of the saddle-point equations and the associated Riemann surface $\tilde{\Sigma}$ it is useful to develop an intuitive picture anticipating some of the key features of the function $t(x)$. We first recall from the results of the previous section that in a field theory vacuum preserving a $U\left(N^{+}\right) \times U\left(N^{-}\right)$gauge symmetry, the field $X$ has the classical eigenvalues $e_{\mu}\left(1, q, \ldots, q^{p-1}\right)$ with a degeneracy of $N^{+}$ for each, and $e_{\mu}\left(q^{p}, q^{p+1}, \ldots, q^{n-1}\right)$ with a degeneracy $N^{-}$. Correspondingly, in the classical limit $g_{s} \rightarrow 0$ the matrix model resolvent will have simple poles at $x \in$ $\left\{e_{\mu}, q e_{\mu}, \ldots, q^{p-1} e_{\mu}\right\}$ with residue $\hat{N}^{+} / \hat{N}$, and at $x \in\left\{q^{p} e_{\mu}, q^{p+1} e_{\mu}, \ldots q^{n-1} e_{\mu}\right\}$ with residue $\hat{N}^{-} / \hat{N}$. This means that in the classical limit the function $t(x)$ defined in (4.7) has only two poles, at $x=e_{\mu}$ and $x=e_{\mu} q^{p}$ with residues $g_{s}\left(\hat{N}^{+}-\hat{N}^{-}\right) e_{\mu}$ and 
$g_{s}\left(\hat{N}^{+}-\hat{N}^{-}\right) q^{p} e_{\mu}$ respectively. Thus, in this simplified classical limit, we can see that the matrix model depends only on one "modulus" $g_{s}\left(\hat{N}^{+}-\hat{N}^{-}\right)$. The nontrivial result which will be argued below is that when the matrix model interactions are turned on $\left(g_{s} \neq 0\right)$, each of these two poles will get smeared into a single branch cut of $t(x)$ and the saddle point condition glues these two cuts together. Importantly, the resulting Riemann surface $\tilde{\Sigma}$ only depends on the modulus $g_{s}\left(\hat{N}^{+}-\hat{N}^{-}\right)$. This modulus is naturally interpreted as the gluino condensate in the $U\left(\left|N^{+}-N^{-}\right|\right)$gauge theory on fractional branes at the end of the duality cascade in the field theory. A similar matrix model interpretation was found in [23] for the duality cascade of the $\hat{A}_{1}$ quiver theory.

\subsection{The general solution}

Let us now turn to the solution of the saddle point equation which will determine the Riemann surface $\tilde{\Sigma}$. We will keep the discussion in this section general and focus on generic deformations $V(x)$ satisfying the requirement (2.1) for generic $n$ with $q^{n}=1$. Later, we will make all of this explicit for the cases $n=2$ with a cubic deformation, and $n>2$ with a quadratic mass deformation.

¿From the saddle point equation (4.8) it is clear that $x$ is a multi-valued function on $\tilde{\Sigma}$ since it jumps by a phase factor $q=e^{2 \pi i / n}$ at points where the cuts are identified. However, this means that $u=x^{n}$ is single-valued on $\tilde{\Sigma}$. So the saddle-point solution of the matrix model has led us to a Riemann surface which admits two meromorphic functions $t$ and $u$. Both $t$ and $u$ have poles at $u=\infty$ and zeros at $u=0$. Furthermore, since $\tilde{\Sigma}$ is covered once by the $x$-plane with cuts, it is an $n$-fold cover of the complex $u$-plane with an $n$-fold branch cut joining all the sheets and running between $u=0$ and $u=\infty$. There are additional cuts $\tilde{\mathcal{C}}_{a}$, the image of each $\mathcal{C}_{a}$, which join adjacent pairs of sheets in the cover.

There is a theorem that if $t$ and $u$ are two meromorphic functions on a Riemann surface then there exists a polynomial function in two variables such that ${ }^{9}$

$$
\tilde{F}(t, u)=0 .
$$

In the classical limit, where the cuts $\tilde{\mathcal{C}}_{a}$ collapse into poles we must recover the singular classical curve $\Sigma(2.22), F(t, u)=0$. It is useful to write

$$
\tilde{F}(t, u)=F(t, u)+\delta(t, u)
$$

where $\delta(t, u)$ is the quantum correction. This correction is constrained by the behaviour of $t$ at $u=0$ and $u=\infty$. Note that due to the branch points at both these

\footnotetext{
${ }^{9}$ For example, in Farkas and Kra [28] Proposition IV.11.6.
} 
points a good coordinate is $x$ rather than $u$ itself. Since $\omega(x)=\mathcal{O}(1 / x)$ for large $x$, we have

$$
t \underset{x \rightarrow \infty}{=} f(x)+\mathcal{O}(1 / x) .
$$

Correspondingly for small $x$ we have

$$
t \underset{x \rightarrow 0}{=} \mathcal{O}(x) .
$$

These two conditions constrain $\delta(t, u)$ to have the form

$$
\delta(t, u)=\sum_{\substack{b \geq 0, a \geq 1 \\ n a+\ell b<(n-1) \ell}} \gamma_{a b} u^{a} t^{b}
$$

The parameters $\left\{\gamma_{a b}\right\}$ are moduli for the solution of the saddle-point equation. One can count the number of these moduli, they number precisely the number of singularities $g$ of $\mathbf{X}$ in (2.29); this is also the (generic) genus of the curve $\tilde{\Sigma}$. In other words, there is a modulus for each singularity of the classical curve $\Sigma$. The picture is now clear. A general solution to the saddle-point equation involves a deformation of the classical curve where each singularity at which pairs of the $n$ copies of the complex $u$-plane touch are resolved into branch cuts. The extent of each branch cut is then controlled by a single modulus. This is precisely the quantum geometry advocated in $[6]$.

Notice that each singularity is only associated to a single cut in the deformed geometry. This means that the matrix model geometry can only accommodate a single density for each pair of fractional branes $\mathfrak{R}_{\mu}^{+}$and $\mathfrak{R}_{\mu}^{-}$. Otherwise one would expect, in general, two cuts in the vicinity of each singularity. This conclusion matches precisely the physics of the RG cascade. The basic point is the following. Suppose that in the vacuum of the field theory $N_{\mu}^{+}>N_{\mu}^{-}$, so there is a net number of $\mathfrak{R}_{\mu}^{+}$fractional branes. In this case, the low energy theory after the cascade should involve a $U\left(N_{\mu}^{+}-N_{\mu}^{-}\right)$gauge group which confines. The matrix model has a single density for the net number of $\mathfrak{R}_{\mu}^{+}$branes and, in particular, there is only one glueball field. Conversely, if $N_{\mu}^{+}<N_{\mu}^{-}$then after the RG cascade a $U\left(N_{\mu}^{-}-N_{\mu}^{+}\right)$gauge group is left which confines. But once again there is only a single glueball field.

So the message from the matrix model is in our vacuum we should only take the net number of fractional branes

$$
N_{\mu} \equiv N_{\mu}^{+}-N_{\mu}^{-}
$$

to infinity in the matrix model. If $N_{\mu}>0(<0)$ then these represent $\mathfrak{R}_{\mu}^{+}\left(\mathfrak{R}_{\mu}^{-}\right)$branes. The remaining $\min \left(N_{\mu}^{+}, N_{\mu}^{-}\right)$fractional branes come as $\mathfrak{R}_{\mu}^{+} \oplus \mathfrak{R}_{\mu}^{-} \simeq \mathfrak{R}^{(n)}\left[u_{\mu}, 0,0\right]$ and can move off into the bulk of $\mathbf{X}$. As advocated in $[5,6]$, these branes should be treated 
as probes in the matrix model calculation. This makes sense since these degrees-offreedom have a moduli space and so we cannot integrate out the fluctuations around them: they remain as probes which feel the quantum geometry deformed by the $\left|N_{\mu}^{+}\right|$ fractional branes. The important conclusion from $[5,6]$ is is that the dynamics of the matrix model can be considered in isolation: there is no "back-reaction" from the probes. The effect of the fractional branes is to change the geometry felt by the branes in the bulk.

Given that there will be only one type of fractional brane in the matrix model from each pair depending upon whether $N_{\mu}^{+} \gtrless N_{\mu}^{-}$, we still have to interpret the single cut joining the two sheets in terms of a density of eigenvalues. Recall that in the classical limit the singularity in question is located at $u=u_{\mu}$. After the deformation, the singularity opens up into a cut whose image in the cut $x$-plane involves two cuts $\mathcal{C}_{\mu}$ and $q^{p_{\mu}} \mathcal{C}_{\mu}$. From this we can reconstruct the density for the fractional. Classically, the eigenvalues are $q^{j-1} e_{\mu}, j=1, \ldots, p_{\mu}$ for $\mathfrak{R}_{\mu}^{+}$and $j=p_{\mu}+1, \ldots, n$ for $\mathfrak{R}_{\mu}^{-}$. In the matrix model these must spread out onto $p_{\mu}$ and $n-p_{\mu}$ cuts, respectively. Let us suppose $N_{\mu}^{+}>N_{\mu}^{-}$, then in order to explain the analytic structure of $t$ it must be that the $p_{\mu}$ cuts are rotational translates of the form $q^{j-1} \mathcal{C}_{\mu}, j=1, \ldots, p_{\mu}$. In addition, the densities along these cuts as measured by the discontinuities in $\omega(x)$ are all equal. This is natural since these cuts are associated to $\mathfrak{R}_{\mu}^{+}$whose eigenvalues are $q^{j-1} e_{\mu}, j=1, \ldots, p_{\mu}$. This means that $t$ defined in (4.7) is left with two cuts at $\mathcal{C}_{\mu}$ and $q^{p_{\mu}} \mathcal{C}_{\mu}$ which are then glued together by the saddle-point equation (4.8). The whole argument can repeated in terms of $\mathfrak{R}_{\mu}^{-}$fractional branes if $N_{\mu}^{+}<N_{\mu}^{-}$.

It follows that if $\mathcal{A}_{\mu}$ is a contour surrounding the cut $\tilde{\mathcal{C}}_{\mu}$ on the curve $\tilde{\Sigma}$ then

$$
S_{\mu} \equiv g_{s} \hat{N}_{\mu}=g_{s}\left(\hat{N}_{\mu}^{+}-\hat{N}_{\mu}^{-}\right)=-\frac{1}{2 n \pi} \oint_{\mathcal{A}_{\mu}} \frac{t d u}{u}
$$

Note that $S_{\mu}$ is positive (negative) for $N_{\mu}^{+}>N_{\mu}^{-}\left(N_{\mu}^{+}<N_{\mu}^{-}\right)$. The quantities $\left\{\tilde{S}_{\mu}\right\}$ and $\left\{\gamma_{a b}\right\}$ form two bases for the moduli space of the curve. The quantity $S_{\mu}$ is interpreted as the glueball field of the $U\left(\left|N_{\mu}\right|\right)$ gauge group factor that confines in the IR.

The final problem remaining is to fix the moduli of the curve in terms of the underlying coupling of the theory. This is done by extremizing the glueball superpotential. The form of this superpotential can be deduced from earlier work [7,21,29]. Recall that the glueball superpotential can only depend on one glueball field for each fractional brane pair. This motivates the expression

$$
W=\sum_{\mu=1}^{g} N_{\mu} \frac{\partial \mathcal{F}_{0}}{\partial \tilde{S}_{\mu}}-2 \pi i \tau \sum_{\mu=1}^{g} \hat{p}_{\mu} S_{\mu}
$$


where we have defined

$$
\hat{p}_{\mu}= \begin{cases}p_{\mu} & N_{\mu}^{+}>N_{\mu}^{-} \\ p_{\mu}-n & N_{\mu}^{+}<N_{\mu}^{-} .\end{cases}
$$

Here, $\mathcal{F}_{0}$ is the first term, "genus zero", in the expansion of the free-energy of the matrix model: $\ln \mathcal{Z}=\sum_{g=0}^{\infty} \mathcal{F}_{g} g_{s}^{2 g-2}$. We have already seen that $S_{\mu}$ can be expressed as an integral around the contour $\mathcal{A}_{\mu}$ which surrounds the cut near $u=u_{\mu}$. The first term in (4.16) can also be expressed in terms of a contour integral on $\tilde{\Sigma}$. It is the variation of the genus zero free-energy (times $g_{s}^{-1}$ ) upon transporting $\left|\hat{p}_{\mu}\right|$ eigenvalues in from infinity to each of the $\left|\hat{p}_{\mu}\right|$ cuts of $\omega(x)$ in the $x$-plane. This can be expressed as the difference of contour integral of $t d u / u$ along a contour that runs out from infinity along the upper sheet of the cut $\tilde{\mathcal{C}}_{\mu}$ minus one that starts on the lower sheet of the cut $\tilde{\mathcal{C}}_{\mu}$ out to infinity. These two open contours clearly join to form a closed contour which can deformed away from infinity. This contour, which we denote $\mathcal{B}_{\mu}$, is the conjugate cycle to $\mathcal{A}_{\mu}$ on $\tilde{\Sigma}$ that goes down the cut $\mathcal{C}_{\mu}$ back along the lower sheet, up through the branch cut joining 0 to $\infty$ and finally out on the upper sheet to join the starting point. In fact $\left\{\mathcal{A}_{\mu}, \mathcal{B}_{\mu}\right\}$ form a basis for the one-cycles of $\tilde{\Sigma}$. Being careful with the normalization,

$$
\frac{\partial \mathcal{F}_{0}}{\partial \tilde{S}_{\mu}}=-\frac{i}{n} \oint_{\mathcal{B}_{\mu}} \frac{t d u}{u} .
$$

and the glueball superpotential is

$$
W=-\frac{i}{n} \sum_{\mu=1}^{g}\left(\tilde{N}_{\mu} \oint_{\mathcal{B}_{\mu}} \frac{t d u}{u}-\hat{p}_{\mu} \tau \oint_{\mathcal{A}_{\mu}} \frac{t d u}{u}\right) .
$$

Now we consider extremizing $W$ with respect to $S_{\mu}$. ¿From the definition of $S_{\mu}$ in (4.15), the one-form

$$
\omega_{\mu}=-\frac{1}{2 n \pi} \frac{\partial}{\partial \tilde{S}_{\mu}}\left(\frac{t d u}{u}\right)
$$

(taken at constant $u$ ) has the property

$$
\oint_{\mathcal{A}_{\mu}} \omega_{\nu}=\delta_{\mu \nu}
$$

Hence, $\left\{\omega_{\mu}\right\}$ is a normalized basis of differentials of the third kind on $\tilde{\Sigma}$. The critical equations can then be written in terms of the period matrix $\tau_{\mu \nu}$ of $\tilde{\Sigma}$ :

$$
\sum_{\mu=1}^{g} N_{\mu} \oint_{\mathcal{B}_{\mu}} \omega_{\nu}=\sum_{\mu=1}^{g} N_{\mu} \tau_{\mu \nu}=\hat{p}_{\nu} \tau
$$

Since $\tilde{\Sigma}$ has $g$ moduli these $g$ equations are enough to determine $\tilde{\Sigma}$ exactly. 
The equations (4.22) are precisely the condition that $\tilde{\Sigma}$ is an $\sum_{\mu=1}^{g} \hat{p}_{\mu} N_{\mu}$-fold cover of a torus of complex structure $\tau$. In order to prove this we need to find a map from $\tilde{\Sigma}$ to the torus $E_{\tau}$ that covers the latter $\sum_{\mu=1}^{g} \hat{p}_{\mu} N_{\mu}$ times. For a point $p \in \tilde{\Sigma}$, the map is

$$
z(p)=2 \pi i \int_{p_{0}}^{p} \sum_{\mu=1}^{g} N_{\mu} \omega_{\mu} \bmod 2 \pi i, 2 \pi i \tau,
$$

where $p_{0}$ is an arbitrary base point. In particular, the glueball superpotential at the critical point is equal to

$$
\begin{aligned}
W^{*} & =-\frac{i}{n} \sum_{\mu=1}^{g}\left(\oint_{\mathcal{A}_{\mu}} d z \oint_{\mathcal{B}_{\mu}} \frac{t d u}{u}-\oint_{\mathcal{B}_{\mu}} d z \oint_{\mathcal{A}_{\mu}} \frac{t d u}{u}\right) \\
& =-2 \pi \operatorname{Res}_{\infty} \frac{f(x) z d x}{x} .
\end{aligned}
$$

In the above, we applied a Riemann bilinear relation and used the fact that $d z=$ $\sum_{\mu} N_{\mu} \omega_{\mu}$ and that $t$ can be replaced with $f(x)$ in the vicinity of its pole at $u=\infty$ $(x=\infty)$.

One remaining issue is to include the bulk branes as probes in the matrix model calculation and to work out the effect of the fractional branes on these probes. We will simply quote the results of [6] here. The classical moduli space of a bulk brane $\mathbf{X}$ is modified by simply replacing the function $f(x)$ by $t(x)$. The second term in (4.7) represents the effect of the fractional branes on the geometry. In other words we replace in 2.20) $F(t, u)$ by the deformation $\tilde{F}(t, u)=F(t, u)+\delta(t, u)$. This defines a quantum deformed moduli space space $\tilde{\mathbf{X}}$ :

$$
u v w=(-1)^{n+1} \tilde{F}(t, u) .
$$

\subsection{The case $q=-1$ with cubic deformation}

As we have noted earlier the theory with $q=-1$ and cubic superpotential (2.3) exhibits a duality cascade in a certain regime of parameter space. This example will allow us to explicitly see in a simple setting, many of the general features argued above. There is one pair of fractional branes so that the deformed curve $\tilde{\Sigma}$ is a torus. Its form will be determined by a deformation of the classical curve (following from (2.12))

$$
F(t, u)=t^{2}-\frac{\epsilon^{2}}{4} u\left(u-a^{2}\right)^{2}=0
$$

In this particular case, we can also derive the quantum deformed curve directly from the matrix model loop equations and since this is interesting we pause to do this. 
The loop equations can be derived by noting that the matrix integral is invariant under generic reparameterizations. In particular consider a variable shift $\delta x_{i} \sim$ $1 /\left(x-x_{i}\right)$ which should leave invariant the integral Eq. (4.2). Following the general algebraic manipulations shown in Appendix A we are led to the following condition (for any finite $\hat{N}$ ):

$$
\left\langle t(x)^{2}\right\rangle=\left\langle\frac{1}{4} x^{2} V^{\prime}(x)^{2}+g_{s} \sum_{i} x^{2}\left(V^{\prime}\left(x_{i}\right)-V^{\prime}(x)\right)\left[\frac{1}{x-x_{i}}-\frac{1}{x+x_{i}}\right]\right\rangle .
$$

In the large- $\hat{N}$ limit, when all averages are dominated by the saddle-point configuration of the matrix integral, we can treat this equation as an ordinary algebraic equation for $t$. The first point to note here is that, when $V(x)$ is an odd polynomial, $t(x)$ is completely determined by a polynomial function on the right hand side of Eq. (4.27). (If $V(x)$ includes even powers, this is no longer true; the right hand side is non-analytic and one also needs to consider higher loop equations.) But we are interested precisely in the cubic polynomial deformations Eq. (2.3), and in the large- $\hat{N}$ limit

$$
t^{2}=\frac{1}{4} \epsilon^{2} x^{2}\left(x^{2}-a^{2}\right)^{2}+2 x^{2} \epsilon \int_{\mathcal{C}} \rho(x) d x .
$$

where on the right hand side we encounter a constant (times $x^{2}$ ) which is the first moment of the eigenvalue distribution. Now we can clearly see how this is related to the deformed (classical) geometry Eq. (2.12) which was the classical moduli space of the field theory. Identifying the variable $u$ in Eq. (2.12) with $x^{2}$ in Eq. (4.28), the above equation reduces to

$$
\tilde{F}(t, u)=t^{2}-\frac{\epsilon^{2}}{4} u\left(u-a^{2}\right)^{2}+\gamma u=0 .
$$

where $\gamma$ is the constant determined by the first moment of the eigenvalue distribution. This is precisely the quantum deformed curve (4.10). The effect of the deformation is to split the double zero at $u=a^{2}$. Thus the function $t$ lives on the two-sheeted complex $u$-plane with two branch cuts. The associated Riemann surface is a torus. It now remains to show how the deformation $\gamma$ will depend on gauge theory parameters. This can be obtained directly by expressing the hyperelliptic curve (4.29) above in Weierstrass form as we show below.

It is also instructive to see how all this follows from the saddle point equation (4.8) for $t(x)$. In this case $t(x)$ has two branch cuts, centred at $x= \pm a$ and is defined as

$$
t(x)=-\frac{1}{2} \epsilon x\left(x^{2}-a^{2}\right)-S[x \omega(x)+x \omega(-x)] .
$$

This in turn implies that $t(x)=-t(-x)$ and hence the two branch cuts are images of each other. The saddle point equation provides a gluing condition on these two 

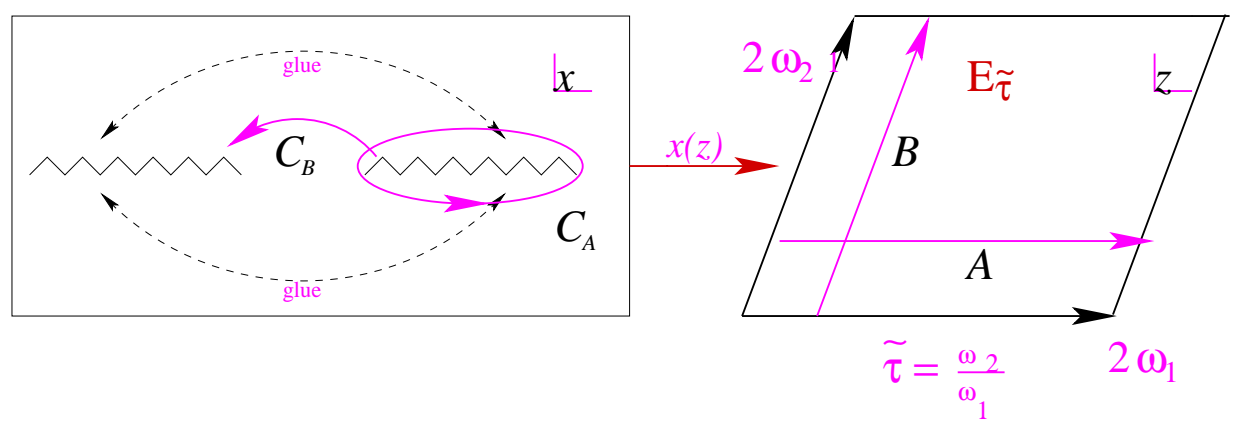

Figure 1: The branch cuts of $t(x)$ and the gluing conditions on them lead to a parameterization in terms of a torus $E_{\tilde{\tau}}$ with complex structure parameter $\tilde{\tau}$.

cuts as illustrated in Fig. 1. The gluing conditions on the two cuts imply that we should think of the Riemann surface as the complex plane with a handle which is a torus. To see this torus explicitly and connect it to gauge theory parameters we first look for an elliptic or Weierstrass parameterization of the problem. (This is exactly the problem that was solved in [7].) In this parameterization the torus of complex structure $\tilde{\tau}$, which we denote $E_{\tilde{\tau}}$, is realized as the fundamental parallelogram in the complex $z$-plane with sides $2 \omega_{1}$ and $2 \omega_{2}$ with opposite identification. Its complex structure is $\tilde{\tau}=\omega_{2} / \omega_{1}$. There is a natural map $x(z)$ from the torus $E_{\tilde{\tau}}$ to the two-cut complex $x$-plane, which must satisfy the conditions:

$$
\begin{array}{lll}
A-\text { cycle shift }: & z \rightarrow z+2 \omega_{1}: \quad x(z) \rightarrow x(z) ; \quad t(x(z)) \rightarrow t(x(z)), \\
B-\text { cycle shift }: & z \rightarrow z+2 \omega_{2}: \quad x(z) \rightarrow-x(z) ; \quad t(x(z)) \rightarrow t(x(z)) .
\end{array}
$$

Hence $x(z)$ is quasi-elliptic, while $t(x(z))$ is an elliptic function on the torus $E_{\tilde{\tau}}$. Both these functions can be determined precisely in terms of the torus variables, from the (quasi)periodicity conditions above and the large- $x$ asymptotics determined by Eq. (4.30). We find,

$$
x(z)=A \sqrt{\wp(z)-e_{1}(\tilde{\tau})} ; \quad t=-\frac{\epsilon}{4} A^{3} \wp^{\prime}(z) ; \quad A=-i a \sqrt{\frac{2}{3}}\left[e_{1}(\tilde{\tau})\right]^{-\frac{1}{2}} .
$$

Here we have used standard notation, $e_{i}(\tilde{\tau})=\wp\left(\omega_{i}\right)$ is the Weierstrass function evaluated at one of the three half-periods $\omega_{1}, \omega_{2}$ and $\omega_{3}=\omega_{1}+\omega_{2}$. The constants of proportionality are uniquely determined by the large- $x$ asymptotics required by Eq. (4.30), which maps to the behaviour in the vicinity of the pole at $z=0$. Now, since

$$
u=x^{2}(z)=A^{2}\left(\wp(z)-e_{1}\right),
$$

as anticipated in our general arguments in the previous section, $u$ and $t$ will satisfy an algebraic relation which in this case follows from the basic differential equation satisfied by the Weierstrass function,

$$
\wp^{\prime 2}(z)=4\left(\wp(z)-e_{1}\right)\left(\wp(z)-e_{2}\right)\left(\wp(z)-e_{3}\right) .
$$


This leads precisely to the algebraic equation (4.7) with

$$
\gamma=\frac{\epsilon^{2} a^{4}}{36}\left[\frac{e_{3}(\tilde{\tau})-e_{2}(\tilde{\tau})}{e_{1}(\tilde{\tau})}\right]^{2} .
$$

Indeed, we could have deduced this directly from Eq. (4.29) by going to an elliptic parameterization, but it is useful to see how the loop equation approach matches up with the saddle point approach.

The relation between the modular parameter $\tilde{\tau}$ of the torus to gauge theory parameters emerges upon extremizing the glueball superpotential of the theory. The glueball superpotential depends on the following period integrals (4.15) which can be explicitly evaluated in terms of the modular parameter $\tilde{\tau}$

$$
S=-\frac{1}{4 \pi} \oint_{\mathcal{A}} \frac{t d u}{u}=\frac{1}{2 \pi i} \frac{d h(\tilde{\tau})}{d \tilde{\tau}}
$$

and

$$
\frac{\partial \mathcal{F}_{0}}{\partial S}=-\frac{i}{2} \oint_{\mathcal{B}} \frac{t d u}{u}=\tilde{\tau} \frac{d h(\tilde{\tau})}{d \tilde{\tau}}-h(\tilde{\tau})
$$

where

$$
h(\tilde{\tau})=\epsilon a^{3} \sqrt{\frac{2}{27 e_{1}(\tilde{\tau})}} .
$$

As we saw from our general arguments, extremizing the glueball superpotential Eq. (4.16), written explicitly in terms of the expressions above, fixes the complex structure parameter of the torus in terms of the gauge theory coupling in a simple way

$$
\tilde{\tau}=\frac{\tau}{\left|N^{+}-N^{-}\right|}
$$

This is what we obtain from (4.22) by noting that the single element of the period matrix of a torus is its complex structure parameter.

Finally we have the critical value of the glueball superpotential, which can be determined explicitly using the above expressions or directly from Eq. (4.24)

$$
W^{*}=\left|N^{+}-N^{-}\right| \epsilon a^{3} \sqrt{\frac{2}{27 e_{1}(\tilde{\tau})}} .
$$

where $\tilde{\tau}=\tau /\left|N^{+}-N^{-}\right|$. The superpotential has a nontrivial expansion in (fractional) instantons when $\operatorname{Im}(\tilde{\tau}) \gg 1$ and vanishes when $N^{+}=N^{-}$. This is in line with our expectation that in the absence of fractional branes the low-energy theory is the Klebanov-Witten conifold SCFT. Note also that the deformation parameter $\gamma$ also vanishes in this case (this follows from the fact that both $e_{2}(\tilde{\tau})$ and $e_{3}(\tilde{\tau})$ approach 

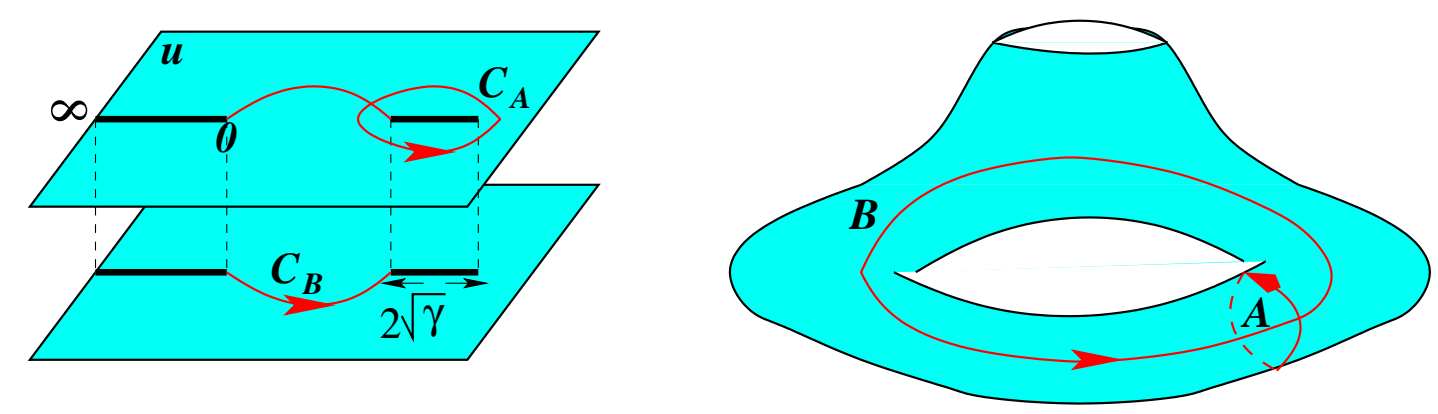

Figure 2: The one-cycles on the $u$-plane which lift to the compact 3-cycles of the CalabiYau geometry.

the same constant value in the $\operatorname{limit} \operatorname{Im}(\tilde{\tau}) \rightarrow \infty)$. An important feature of the IR gauge theory is reflected in this critical superpotential, namely that it is not pure $\mathcal{N}=1$ SYM. It has massive modes which are not decoupled from the $\mathcal{N}=1$ gauge multiplet. The theory does reduce to the pure $\mathcal{N}=1$ gauge theory in a decoupling limit $\hat{\tau} \rightarrow i \infty$ keeping fixed the dynamical scale $a \epsilon^{1 / 3} \exp (2 \pi i \tilde{\tau} / 3)$. This is also apparent in the structure of the deformed geometry.

The full deformed Calabi-Yau geometry (Fig. 2) is obtained from the matrix model Riemann surface adding uvw to the right hand side of Eq. (1.29). In this deformed Calabi-Yau geometry:

$$
t^{2}=-u v w+\frac{\epsilon^{2}}{4} u\left(u-a^{2}\right)^{2}+u \gamma
$$

the isolated conifold singularity at $u=a^{2}$ has been replaced by a 3-sphere of a finite size controlled by $\gamma$ which in turn is determined in terms of gauge theory parameters by Eqs. (4.35) and (4.39). This is the deformation seen in the geometric dual at the endpoint of the cascade.

It is also interesting to compare and contrast the loop equation (the matrix model Riemann surface) for the $\hat{A}_{1}$ quiver theory and that for our theory with $q=-1$. Recalling the result of [23], the infrared physics at the endpoint of the cascade in the $\hat{A}_{1}$ quiver theory is controlled by a matrix model curve:

$$
y^{2}=x^{2}+\mu^{2},
$$

which is the complex $x$-plane with a single branch cut. There are two one-cycles, one of which is compact (the contour enclosing the cut) while its dual cycle is noncompact (running from a branch point to infinity). This lifts to the deformed conifold geometry $y^{2}=u v+x^{2}+\mu$ with one three-cycle of finite size. This is, of course, what one expects for the pure $\mathcal{N}=1$ SUSY Yang-Mills theory in the IR. 
In contrast, as we have seen in Eq. (4.29) the theory under investigation leads to the two-cut complex $u$-plane with two compact cycles. One of these corresponds to the contour enclosing the cut between $u=a^{2}+2 i \sqrt{\gamma} / \epsilon$ and $u=a^{2}-2 i \sqrt{\gamma} / \epsilon$. The dual cycle is given by the contour running from $u=0$ to $u=a^{2}+2 i \sqrt{\gamma} / \epsilon$, moving down to the second sheet and running back to $u=0$ (which is the tip of the second cut extending from $u=0$ to $u=\infty)$. In a certain limit $(a \rightarrow \infty)$ where one of these cycles becomes noncompact we recover the physics of the pure $\mathcal{N}=1 \mathrm{SYM}$ in the infrared. This is also the decoupling limit for the adjoint fields in Eq. (3.3).

\subsection{The case $n>2$ with quadratic deformation}

This case with quadratic superpotential (3.6) allows us to embed the duality cascade within a relevant deformation of the UV-finite four-dimensional SCFT. We will now determine the quantum deformed geometry associated to this theory. Recall that classically, the Higgs branch moduli space of this theory is described by the algebraic curve Eq. (2.20), uvw $=(-1)^{n+1} F(t, u)$. It has $[n / 2]$ isolated singularities of the conifold type where $v=w=0$ and where the (classical) Riemann surface described by $F(t, u)=0$ becomes singular. Here the classical curve

$$
F(t, u)=\prod_{i=1}^{n}\left(t+\epsilon \frac{q^{2 i} x^{2}}{1-q^{2}}-\epsilon a \frac{q^{i} x}{1-q}\right)=0 .
$$

Note that this curve is only a function of $u=x^{n}$ since it is invariant under $x \rightarrow x q$. The singularities occur at the points $u=u^{(p)}, p<n$ given by Eq. (3.7) where the classical curve above has a double zero. Fractional branes can be located at each of these conifold singularities. In all there are $[n / 2]$ distinct types of fractional brane pairs. The effect of sticking fractional branes at a conifold singularity is to split the double zeroes, resulting in a branch cut that joins a pair of $n$ sheets of the Riemann surface associated to $t$ as a function of $u$. This is exactly what we encountered in the previous example with $n=2$. This deformation leads us to the quantum curve (4.10) which takes the form

$$
\tilde{F}(t, u)=\prod_{i=1}^{n}\left(t+\epsilon \frac{q^{2 i} x^{2}}{1-q^{2}}-\epsilon a \frac{q^{i} x}{1-q}\right)+u \sum_{a=0}^{[(n-3) / 2]} \gamma_{a} t^{a}=0
$$

The $\gamma_{a}$ are the quantum deformation parameters which we would like to determine using the matrix model. They are implicitly functions of the glueball condensate of the field theory. The curve is actually hyper-elliptic since it has the general form

$$
\tilde{F}(t, u)=t^{n}+\left(\frac{\epsilon}{1-q^{2}}\right)^{n} u^{2}+u \sum_{a=0}^{[n / 2]} s_{a} t^{a}=0
$$


where the $s_{a}$ depend on the moduli. In fact, $s_{a}$ is equal to $\gamma_{a}$, for $a=0, \ldots,[(n-3) / 2]$, up to a constant shift, while $s_{a}$, for $a=[(n-1) / 2], \ldots,[n / 2]$ only depend on the parameters in the potential.

Generically when all types of fractional branes are present the curve has genus $[n / 2]$, as each of the singularities gets opened up into a branch cut. However, let us consider the simplest vacuum, which was described in Section 3.2 where fractional branes are present at only one of the conifold singularities. This means that only one cut opens up leaving a genus one Riemann surface, or torus. In this case, we can solve for $t$ and $u$ (and $x$ ) in terms of the Weierstrass parameterization.

The parameterization we seek can be deduced from the saddle-point condition (4.8), which says that the two cuts of $t(x)$ which are images under a rotation by $q$ are now glued together. This adds a handle to the complex $x$-plane giving us a torus (Fig. 2) with a complex structure modulus $\tilde{\tau}$. As explained in Section 4.3, we think of this torus $E_{\tilde{\tau}}$ as the complex $z$-plane modded out by lattice translations. Going around the A-cycle of this torus transforms $x$ by a phase $x(z) \rightarrow q x(z)$ while the B-cycle shift leaves $x(z)$ invariant. Clearly this means that the $u(z)=x^{n}(z)$ is an elliptic function of $z$ (this is true only because $q$ is an $n$-th root of unity). These operations also leave $t(x(z))$ invariant. Along with the large- $x$ asymptotics (which maps to small- $z$ ), this determines the two functions uniquely:

$$
u(z)=A^{n} \cdot \frac{\theta_{1}^{n}\left(\pi z / 2 \omega_{1}-p \pi / n\right)}{\theta_{1}^{n}\left(\pi z / 2 \omega_{1}\right)} ; \quad t(x(z))=B \cdot\left[\wp(z)-\wp\left(2 \omega_{1} p / n\right)\right] .
$$

where the constants $A$ and $B$ are

$$
A=a \cdot \frac{(1+q)}{2} \cdot \frac{\theta_{1}^{\prime}(0)}{\theta_{1}^{\prime}(p \pi / n)} ; \quad B=-\epsilon a^{2} \cdot \frac{\omega_{1}^{2}}{\pi^{2}} \cdot \frac{1+q}{1-q} \cdot \frac{\theta_{1}^{2}(p \pi / n)}{\theta_{1}^{\prime 2}(p \pi / n)}
$$

The modular parameter associated to all the (quasi)elliptic functions appearing in the above expressions is $\tilde{\tau}$, the complex structure parameter of the torus $E_{\tilde{\tau}}$.

$u(z)$ is an elliptic function with an $n$-th order pole at $z=0$ and an $n$-th order zero at $z=2 p \omega_{1} / n$. By determining the coefficients of the singular (and constant) pieces in the Laurent expansion of $u$ around $z=0$, we can rewrite $u(z)$ in terms of the Weierstrass function and its derivatives using well-known results for elliptic functions (Eq. (B.19)). This straightforward (but lengthy) procedure allows us to obtain the algebraic relation between $u$ and $t$. For example for $n=3$ and $p=1$ we find

$$
\begin{aligned}
\tilde{F}(t, u)=t^{3}+[ & \left.\frac{\epsilon}{1-q^{2}}\right]^{3} u^{2}-3 a\left[\frac{\epsilon}{\left(1-q^{2}\right)}\right]^{2} t u \\
& +\frac{a^{3}}{8}\left[\frac{\epsilon}{1-q}\right]^{3} \frac{\theta_{1}^{3}(\pi / 3)}{\theta_{1}^{\prime 3}(\pi / 3)} \wp^{\prime}\left(2 \omega_{1} / 3\right) u=0 .
\end{aligned}
$$




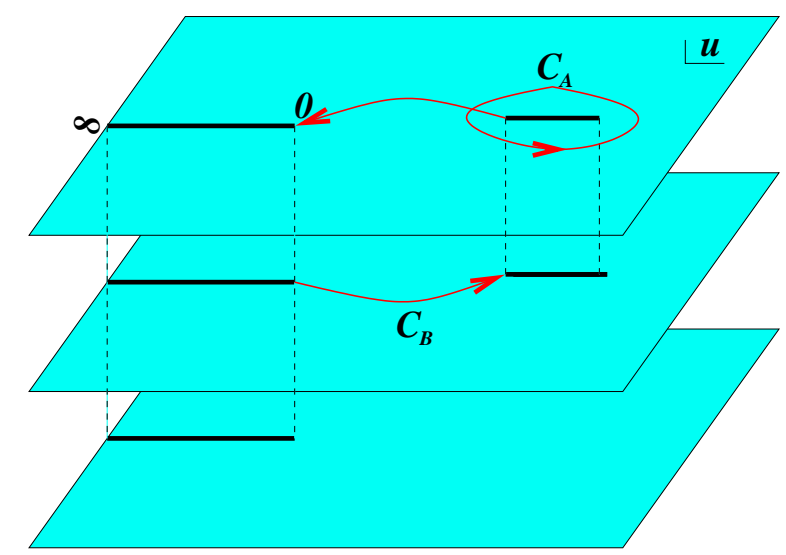

Figure 3: The Riemann surface for $t(u)$ in the case $n=3$. The two one-cycles on the $u$-plane lift to compact 3-cycles of the Calabi-Yau space.

This quantum curve has all the properties that we anticipated in and below Eq. (4.45). (For the $n=3$ case, where $[n / 2]=1$, there is actually only one conifold singularity.) In particular there is only one quantum deformation parameter which alters the coefficient of the term linear in $u$. As we see below the classical limit-absence of fractional branes - corresponds to taking $\operatorname{Im}(\tilde{\tau})$ to $\infty$. In this limit the quantum deformation that we have computed above indeed approaches the result expected from the classical curve Eq. (2.20). The classical Riemann surface associated to $t(u)$ consists of three sheets joined via the branch cut running from $u=0$ to $u=\infty$. The effect of the quantum deformation is to open up the singularity at $u=a^{3}$ into a square root branch cut that joins two of the three sheets (Fig. 3). The algebraic equation for the deformed Calabi-Yau space is, as before

$$
u v w=(-1)^{n+1} \tilde{F}(t, u)
$$

Once again we can relate the complex structure $\tilde{\tau}$ to the gauge theory coupling by extremizing the glueball superpotential as in (4.22). For a gauge theory vacuum where we have $N^{+}$and $N^{-}$fractional branes of the two different types, we find the relation $^{10}$

$$
\tilde{\tau}=\frac{\hat{p} \tau}{N^{+}-N^{-}}= \begin{cases}p \tau /\left(N^{+}-N^{-}\right) & N^{+}>N^{-} \\ (n-p) \tau /\left(N^{-}-N^{+}\right) & N^{+}<N^{-}\end{cases}
$$

Hence, the limit where the deformation vanishes i.e. $N^{+}=N^{-}$is when $\operatorname{Im}(\tilde{\tau}) \rightarrow \infty$.

\footnotetext{
${ }^{10}$ This can be explicitly seen by evaluating $S$ and $\partial \mathcal{F}_{0} / \partial S$ which turn out to be $d h(\tilde{\tau}) / d \tilde{\tau}$ and $\tilde{\tau} d h(\tilde{\tau}) / d \tilde{\tau}-h(\tilde{\tau})$ where $h(\tilde{\tau})=2 \epsilon a^{2}[(1+q) /(1-q)] \theta_{1}(p \pi / n) / \theta_{1}^{\prime}(p \pi / n)$. Extremizing the superpotential $(4.16)$ then leads to the desired relation between $\tilde{\tau}$ and the gauge theory parameter.
} 
Finally from (4.24) we have the critical value of the glueball superpotential:

$$
W^{*}=-2\left|N^{+}-N^{-}\right| \epsilon a^{2} \frac{1+q}{1-q} \cdot \frac{\theta_{1}(p \pi / n)}{\theta_{1}^{\prime}(p \pi / n)}
$$

where the modular parameter of the Jacobi theta functions, $\tilde{\tau}$ is determined as above. This value of the effective superpotential coincides with that of the mass deformation of the $\beta$-deformed theory obtained in [7]. This indicates that at least at the level of the holomorphic sector, the low-energy theory at the end of the RG cascade is the mass deformation of the $U\left(\left|N^{+}-N^{-}\right|\right) \beta$-deformed theory (with $\beta=2 \pi / n$ ). Pure $\mathcal{N}=1$ SUSY gauge theory only emerges in a decoupling limit for the extra massive adjoint fields.

\subsection{Relation to periods of the Calabi-Yau geometry}

It is interesting to explicitly see the relation of the matrix model moduli to periods of the Calabi-Yau geometry. We know that a vacuum of the $\mathcal{N}=1$ theory with $U\left(N^{+}\right) \times$ $U\left(N^{-}\right)$gauge symmetry is obtained by taking $N^{+}$fractional D3-branes of one type and $N^{-}$of the second type and placing them at a conifold singularity in the (classical) geometry specified by Eq. (2.20). The general picture is that gaugino condensation in the infrared theory leads to a geometric dual where the $S^{2}$ is replaced by a deformed $S^{3}$ without any branes. For the B-model topological string in this deformed geometry, the genus zero prepotential term is determined by period integrals of the holomorphic $(3,0)$ form. These period integrals of the deformed Calabi-Yau geometry should

precisely be the matrix model integrals of the meromorphic one-form over the $\mathcal{A}$ and $\mathcal{B}$ cycles of the Riemann surface. We show that this is indeed the case.

First, in the simplest case with $q=-1$, consider the holomorphic $(3,0)$ form associated to the geometry (2.12),

$$
\begin{aligned}
\Omega & =d t^{\prime} \wedge d u \wedge d v \wedge d w \delta\left(t^{\prime 2}+u v w-\frac{\epsilon^{2}}{4} u\left(u-a^{2}\right)^{2}+\gamma u\right) \\
& =\frac{d u \wedge d v \wedge d w}{2 \sqrt{u\left[-v w+\frac{\epsilon^{2}}{4}\left(u-a^{2}\right)^{2}+\gamma\right]}} .
\end{aligned}
$$

The geometry has compact 3-cycles over which the period integrals can be evaluated as follows. First one picks an appropriate reality condition on the coordinates (e.g. $v=w^{*}$ assuming that $a$ and $\gamma$ are real, positive). The 3 -cycles can be identified by treating $v$ and $w$ as coordinates on an $S^{2}$ which is fibred over an appropriately chosen interval on the $u$-plane. In this way the period integrals over the compact 3-cycles in the Calabi-Yau, reduce to contour integrals over the branch cuts (Fig. 3) 
of a meromorphic 1-form in the $u$-plane,

$$
\oint_{A, B} \Omega=2 \pi \oint_{\mathcal{A}, \mathcal{B}} \sqrt{\frac{\epsilon^{2}}{4}\left(u-a^{2}\right)^{2}+\gamma} \frac{d u}{\sqrt{u}}=2 \pi \oint_{\mathcal{A}, \mathcal{B}} t \frac{d u}{u},
$$

which are precisely the moduli of the matrix model solution discussed above.

More generally, we can present an argument along the lines of [30]. For the non-compact Calabi-Yau given as the hypersurface in $\mathbf{C}^{4}$,

$$
u v w=(-1)^{n+1} \tilde{F}(t, u)
$$

where $\tilde{F}(t, u)$ is defined as in Eq.(4.10), we think of the Calabi-Yau space $\mathbf{X}$ as a fibration over the $(t, u)$-plane, where the fibre is the curve $u v w=(-1)^{n+1} \tilde{F}$. The holomorphic $(3,0)$ form can be chosen to be

$$
\Omega=\frac{d t \wedge d u \wedge d v}{u v}
$$

This has periods over the three-cycles in $\mathbf{X}$ which reduce (by Cauchy's theorem) to

$$
\int_{D} \frac{d t \wedge d u}{u}
$$

where $D$ is a real two-dimensional domain in the complex $(t, u)$-plane such that $\partial D \subset \tilde{\Sigma}$. Here $\tilde{\Sigma}$ is the Riemann surface $\tilde{F}(t, u)=0$ on which the fibre degenerates. By Stokes' theorem these integrals reduce to

$$
\oint_{\mathcal{A}_{\mu}, \mathcal{B}_{\mu}} \frac{t d u}{u}
$$

integrals of a meromorphic one-form over the one-cycles of the Riemann surface $\tilde{\Sigma}$. These are precisely the moduli $\left(\tilde{S}_{\mu}, \partial \mathcal{F}_{0} / \partial \tilde{S}_{\mu}\right)$ of the matrix model (Eqs. (4.15) and (4.18).)

\section{A Generalization}

Finally, we turn to a more general class of renormalizable deformations of the superconformal field theory (with $q^{n}=1$ ) described by the superpotential

$$
W=\operatorname{Tr}\left[X Y Z-q X Z Y-m_{1} Y Z-m_{2} X Z-m_{3} X Y\right] .
$$

By shifting each field by a multiple of the identity one has the equivalent description

$$
W=\operatorname{Tr}[X Y Z-q X Z Y-2 \sigma X-2 \zeta Y-2 \eta Z],
$$


where

$$
\sigma=\frac{m_{2} m_{3}}{2(q-1)} \quad \zeta=\frac{m_{1} m_{3}}{2(q-1)}, \quad \eta=\frac{m_{1} m_{2}}{2(q-1)} .
$$

This is precisely the deformation considered in $[15,16]$. It completely resolves the orbifold fixed lines leaving behind $(n-1)$ isolated conifold singularities.

As previously it is useful to consider a slightly more general form of the theory by introducing a general superpotential for $X$ :

$$
W=\operatorname{Tr}[X Y Z-q X Z Y-2 \zeta Y-2 \eta Z-V(X)]
$$

We are going to show that this more general deformation can easily be incorporated into our existing formalism by a simple trick. The idea is to "complete the square", in $Y$ and $Z$ by defining

$$
Y=\tilde{Y}-\frac{2 \eta}{q-1} X^{-1}, \quad Z=\tilde{Z}-\frac{2 \zeta}{q-1} X^{-1}
$$

This move changes the superpotential for $X$ to

$$
\tilde{V}(x)=\sum_{j \neq 0 \bmod n}^{\ell} \frac{a_{j}}{j} x^{j}-\frac{4 \zeta \eta}{q-1} x^{-1} .
$$

Our previous formalism now applies with $V$ replaced by $\tilde{V}$. Hence the classical moduli space $\mathbf{X}$ of the Higgs branch is now

$$
u \tilde{v} \tilde{w}=(-1)^{n+1} F_{\tilde{V}}(t, u),
$$

where $F_{\tilde{V}}(t, u)$ is given as in (2.20) but with $f(x)$ defined as in (2.14) with $V(x)$ replaced by $\tilde{V}(x)$ and

$$
\tilde{v}=\frac{1}{n} \operatorname{Tr} \tilde{Y}^{n}, \quad \tilde{w}=\frac{1}{n} \operatorname{Tr} \tilde{Z}^{n}
$$

Since

$$
v=\tilde{v}+\left(\frac{2 \eta}{1-q}\right)^{n} \frac{1}{u}, \quad w=\tilde{w}+\left(\frac{2 \zeta}{1-q}\right)^{n} \frac{1}{u},
$$

we can write the moduli space in terms of the original variables as

$$
u v w=(-1)^{n+1} F_{\tilde{V}}(t, u)+\left(\frac{2 \eta}{1-q}\right)^{n} v+\left(\frac{2 \zeta}{1-q}\right)^{n} w-\left(\frac{2 \zeta \eta}{(1-q)^{2}}\right)^{n} \frac{1}{u} .
$$

It turns out that the right-hand side is a polynomial in $t$ and $u$ since the $\mathcal{O}(1 / u)$ terms cancel.

The classical singularity structure of $\mathbf{X}$ is somewhat different from the case with $\zeta=\eta=0$. The singular line at $u=0$ is now resolved and only isolated conifoldlike singularities remain. In this case the Higgs branch becomes disconnected from 
the Coulomb branch. As before, the isolated singularities are associated to pairs of representations $\mathfrak{R}_{\mu}^{ \pm}$. The matrix model analysis can be followed as in Section 4 to find the quantum deformed geometry. The only difference is that the quantum deformation of the curve is slightly more general with (4.10) replaced by

$$
\delta(t, u)=\sum_{\substack{b \geq 0, a \geq 0 \\ n a+\ell b<(n-1) \ell}} \gamma_{a b} u^{a} t^{b}
$$

For example, in the case with $q=-1$ and with $V(x)=2 \sigma x$, the shifted potential is

$$
\tilde{V}(x)=2 \sigma x+\frac{2 \zeta \eta}{x} .
$$

The classical moduli space (2.20) is

$$
u v w=-t^{2}+\sigma^{2} u+\zeta^{2} v+\eta^{2} w-2 \sigma \zeta \eta
$$

in particular, there is a single singularity at $v=w=0$ and $u=\zeta \eta / \sigma$. The quantum curve $\tilde{\Sigma}$ is

$$
\tilde{F}(t, u)=t^{2}-\sigma^{2} u-\zeta^{2} \eta^{2} \frac{1}{u}+2 \sigma \zeta \eta+\gamma=0
$$

where $\gamma$ is the quantum deformation. This curve is, as expected, a torus. It can be written in Weierstrass form (4.34) by taking

$$
u=B \wp(z), \quad t=\frac{A \wp^{\prime}(z)}{B \wp(z)},
$$

where

$$
A=\left(\frac{g_{3}}{\zeta^{2} \eta^{2}}\right)^{1 / 2}, \quad B=\left(\frac{4 \zeta^{2} \eta^{2}}{\sigma g_{3}}\right)^{1 / 3} .
$$

Here, $g_{2}$ and $g_{3}$ are the Weierstrass invariants defined in (B.6). The quantum deformation in (5.14) is then

$$
\gamma=g_{2}\left(\frac{\sigma \zeta^{4} \eta^{4}}{4 g_{3}^{2}}\right)^{1 / 3}-2 \sigma \zeta \eta
$$

The critical point of the glueball superpotential will set the complex structure of the torus $\tilde{\tau}$ as in (4.39). Replacing $F$ by $\tilde{F}$ in (5.13) then gives the quantum deformed geometry $\tilde{\mathbf{X}}$ as a function of the underlying couplings.

\section{Conclusions}

We have studied relevant deformations of the $\mathcal{N}=1$ SCFT related to $\mathcal{N}=4$ theory by the exactly marginal $\beta$-deformation. We have seen how at intermediate energy 
scales, certain vacua of the theory (at weak gauge coupling) can have a description in terms of an $\mathcal{N}=1$ theory undergoing a Klebanov-Strassler duality cascade which terminates in an IR theory with a mass gap and gaugino condensation. We understood all of this rather precisely in the language of the Dijkgraaf-Vafa matrix model which allowed us to compute the quantum deformed moduli space seen by a probe D-brane at the end of the cascade. Specifically we were able to compute exactly the deformation parameters as functions of the microscopic parameters of the gauge theory. A natural question arising in the context of these theories, which we hope to address in future work, is what is the supergravity/string theory dual for these theories. In the UV the field theory (at large- $N$ ) is (super)conformal and is dual to the near horizon geometry $A d S_{5} \times S^{5} / \Gamma$ ( $\Gamma$ being the orbifold action) of D-branes at the $\mathbf{C}^{3} / \mathbf{Z}_{n} \times \mathbf{Z}_{n}$ orbifold with discrete torsion. ${ }^{11}$ The discrete torsion should be encoded in the boundary conditions for massless twisted states of the orbifold. As noted in [6] the relevant deformations we have studied correspond to turning on certain fields in the twisted sector. This deformation should cause the AdS geometry to smoothly match on to a Klebanov-Strassler type geometry that describes the cascade phenomenon in the IR field theory. It would be extremely interesting to obtain such a SUGRA flow. Of course, from the point of view of the field theory results we should also expect to find a flow interpolating between $A d S_{5} \times S^{5} / \Gamma$ and $A d S_{5} \times T^{(1,1)}$ which is the conifold theory of [24].

Acknowledgements: We would like to thank N. Dorey, G. Policastro and F. Quevedo for discussions.

\section{Appendix A: Matrix model loop equations}

We now outline how the quadratic loop equations follow from the integral (4.2). Performing a variable change $\delta x_{i}=\frac{\epsilon}{x-x_{i}}$, the invariance of the integral under general variable changes leads to the condition

$$
\begin{aligned}
& \left\langle\sum_{i} \frac{1}{\left(x-x_{i}\right)^{2}}+\sum_{i \neq j} \frac{1}{\left(x_{i}-x_{j}\right)}\left[\frac{1}{x-x_{i}}-\frac{1}{x-x_{j}}\right]-\sum_{i \neq j} \frac{1}{\left(x_{i}-q x_{j}\right)}\left[\frac{1}{x-x_{i}}-\frac{q}{x-x_{j}}\right]\right\rangle \\
& =\left\langle\frac{\hat{N}}{S} \sum_{i} \frac{V^{\prime}\left(x_{i}\right)}{x-x_{i}}\right\rangle .
\end{aligned}
$$

\footnotetext{
${ }^{11}$ When $n$ is large and $q$ is close to 1 , we can also think of this theory as a small deformation of the $\mathcal{N}=4$ theory and consequently its SUGRA dual corresponds to switching on a non-normalizable supergravity mode in the $A d S_{5} \times S^{5}$ background. The relation between the two descriptions has been discussed in [17].
} 
Now, the identities

$$
\frac{1}{\left(x-x_{i}\right)\left(x_{i}-q x_{j}\right)}=\frac{1}{\left(x-x_{i}\right)\left(x-q x_{j}\right)}+\frac{1}{\left(x-q x_{j}\right)\left(x_{i}-q x_{j}\right)}
$$

and

$$
\frac{1}{\left(x-x_{i}\right)\left(q x_{i}-x_{j}\right)}=\frac{1}{\left(x-x_{i}\right)\left(q x-x_{j}\right)}+\frac{q}{\left(q x-x_{j}\right)\left(q x_{i}-x_{j}\right)}
$$

can be used recursively along with Eq.(A.1) to obtain the following condition on the resolvent function

$$
\left.\sum_{k=0}^{n-1}\left\langle\left[q^{k} \omega\left(x q^{k}\right)\right\rangle-q^{k+1} \omega\left(x q^{k+1}\right)\right]^{2}\right\rangle=\frac{2}{S} \frac{1}{\hat{N}} \sum_{i=1}^{\hat{N}} \sum_{k=0}^{n-1} q^{2 k}\left\langle\frac{V^{\prime}\left(x_{i}\right)}{x q^{k}-x_{i}}\right\rangle .
$$

We can rewrite this as

$$
\begin{aligned}
& \sum_{k=0}^{n-1}\left\langle\left[q^{k} x \omega\left(x q^{k}\right)-q^{k+1} x \omega\left(x q^{k+1}\right)+\frac{1}{S} f\left(q^{k+1} x\right)\right]^{2}\right\rangle=\frac{1}{S^{2}} \sum_{k=0}^{n-1} f^{2}\left(q^{k+1} x\right)+ \\
& +\frac{2}{S} \frac{1}{\hat{N}} \sum_{i=1}^{\hat{N}} \sum_{k=0}^{n-1} q^{2 k} x^{2}\left\langle\frac{V^{\prime}\left(x_{i}\right)-V^{\prime}\left(x q^{k}\right)}{x q^{k}-x_{i}}\right\rangle .
\end{aligned}
$$

In terms of the function $t(x)$ defined in Eq.(4.7) the quadratic loop equation simplifies

$$
\sum_{k=1}^{n}\left\langle t^{2}\left(q^{k} x\right)\right\rangle=\sum_{k=1}^{n} f^{2}\left(q^{k} x\right)+S \frac{2}{\hat{N}} \sum_{i=1}^{\hat{N}} \sum_{k=0}^{n-1} q^{2 k} x^{2}\left\langle\frac{V^{\prime}\left(x_{i}\right)-V^{\prime}\left(x q^{k}\right)}{x q^{k}-x_{i}}\right\rangle .
$$

For $n=2$ and a perturbation that satisfies the condition (2.3) the right hand side is a polynomial and the quadratic loop equation is sufficient to determine $t(x)$ in the large- $\hat{N}$ limit when the loop equation can be treated as an ordinary algebraic equation. For $n>2$ the loop equation above does not determine $t(x)$.

For $n>2$ we need the higher loop equations. These are more difficult to derive directly. However, we can deduce their form by noting an interesting connection to the loop equations for the matrix model associated to the $A_{n-1}$ quiver theory. (Here we mean the $\mathcal{N}=2$ quiver theory with polynomial superpotential deformations for the $n$ adjoint chiral multiplets. These softly break $\mathcal{N}=2$ supersymmetry to $\mathcal{N}=1$.) The higher order loop equations for the $A_{n-1}$ quiver matrix model were derived in [33] using free boson techniques. The quadratic $A_{n-1}$ loop equations derived by them (see Eq.(2.64) of [33]) is in fact identical to our Eq. (A.5) provided we identify the $k$-th resolvent of the quiver model $\omega_{k}(x)$ with $q^{k} \omega\left(q^{k} x\right)$ in our theory. We need to make a similar identification of the deforming polynomials. Basically, the different factors in the quiver are constrained to be the same, up to a phase factor $q$ between 
adjacent factors in the quiver. This is not a coincidence. It is related to the fact that the theory on D-branes at the orbifold $\mathbf{C}^{3} / \mathbf{Z}_{n} \times \mathbf{Z}_{n}$ with discrete torsion can be obtained by the "orbifolding of an orbifold", [15] (i.e. $\mathbf{Z}_{n}$ orbifold of $\mathbf{C} \times \mathbf{C} / \mathbf{Z}_{n}$ ). The phase factor $q$ between adjacent factors of the quiver represents the single unit of discrete torsion. The $n$-th order loop equations for our theory can be obtained by applying this prescription to the results of [33]. It would also be interesting to apply these ideas to the derivation of loop equations from anomaly chains as done in [34].

\section{Appendix B: Some Properties of Elliptic Functions}

In this appendix we provide some useful - but far from complete - details of elliptic functions and their near cousins. For definitions and a more complete treatment, we refer the reader to one of the textbooks, for example [31]. An elliptic function $f(u)$ is a function on the complex plane, periodic in two periods $2 \omega_{1}$ and $2 \omega_{2}$. We will define the lattice $\Gamma=2 \omega_{1} \mathbb{Z} \oplus 2 \omega_{2} \mathbb{Z}$ and define the basic period parallelogram as

$$
\mathcal{D}=\left\{u=2 \mu \omega_{1}+2 \nu \omega_{2}, 0 \leq \mu<1,0 \leq \nu<1\right\}
$$

The complex structure of the torus defined by identifying the edges of $\mathcal{D}$ is

$$
\tau=\omega_{2} / \omega_{1}
$$

and we also define

$$
q=e^{i \pi \tau}
$$

\section{B.1 The Weierstrass function}

The archetypal elliptic function is the Weierstrass $\wp(u)$ function. It is an even function which is analytic throughout $\mathcal{D}$, except at $u=0$ where it has a double pole:

$$
\begin{aligned}
& \wp(u)=\frac{1}{u^{2}}+\sum_{k=1}^{\infty} c_{k+1} u^{2 k}, \\
& c_{2}=\frac{g_{2}}{20}, \quad c_{3}=\frac{g_{3}}{28}, \quad c_{k}=\frac{3}{(2 k+1)(k-3)} \sum_{j=2}^{k-2} c_{j} c_{k-j} \quad k \geq 4 .
\end{aligned}
$$

The Weierstrass function satisfies the fundamental identity

$$
\left(\frac{d}{d u} \wp(u)\right)^{2}=4 \wp(u)^{3}-g_{2} \wp(u)-g_{3}
$$

which defines the Weierstrass invariants $g_{2,3}=g_{2,3}\left(\omega_{\ell}\right)$ associated to the torus. If the values of $\wp\left(\omega_{i}\right)$ be $e_{i},(i=1,2,3)$, then $\left\{e_{i}\right\}$ are roots of the equation $4 t^{3}-g_{2} t-g_{3}=0$. 
Here $\omega_{3}=\omega_{1}+\omega_{2}$. From the formulae connecting roots of equations with their coefficents, it follows that

$$
e_{1}+e_{2}+e_{3}=0, \quad e_{1} e_{2}+e_{2} e_{3}+e_{3} e_{1}=-\frac{1}{4} g_{2}, \quad e_{1} e_{2} e_{3}=\frac{1}{4} g_{3} .
$$

\section{B.2 The Weierstrass zeta function}

We are also interested in other functions which are only quasi-elliptic. First we have $\zeta(u)$. It is an odd function with the quasi-elliptic property:

$$
\zeta\left(u+2 \omega_{\ell}\right)=\zeta(u)+2 \zeta\left(\omega_{\ell}\right) .
$$

Its derivative gives minus the Weierstrass function

$$
\wp(u)=-\zeta^{\prime}(u) \text {. }
$$

It follows that $\zeta(u)$ has a simple pole at $u=0$. Useful identities are

$$
\begin{aligned}
& \omega_{2} \zeta\left(\omega_{1}\right)-\omega_{1} \zeta\left(\omega_{2}\right)=\frac{\pi i}{2}, \\
& \zeta\left(\omega_{1}+\omega_{2}\right)=\zeta\left(\omega_{1}\right)+\zeta\left(\omega_{2}\right) .
\end{aligned}
$$

The (quasi)-elliptic functions evaluated at rational multiples of their periods satisfy special relations. For example, one useful identity that we need is

$$
\zeta\left(2 \omega_{i} / 3\right)-\frac{2}{3} \zeta\left(\omega_{i}\right)=\left[\frac{1}{3} \wp\left(2 \omega_{i} / 3\right)\right]^{1 / 2}
$$

\section{B.3 The Jacobi theta functions}

We are also interested in the Theta functions $\theta_{i}(x \mid \tau)$, or $\theta_{i}(x, q), i=1,2,3,4$. They are also quasi-elliptic functions on $\mathcal{D}$ when $x=\pi u / 2 \omega_{1}$. Each of them satisfies the heat equation

$$
\pi i \frac{\partial^{2} \theta_{i}(x \mid \tau)}{\partial x^{2}}+4 \frac{\partial \theta_{i}(x \mid \tau)}{\partial \tau}=0
$$

They are related to the previous functions; for instance,

$$
\zeta(u)-\frac{\zeta\left(\omega_{1}\right)}{\omega_{1}} u=\left.\frac{\pi}{2 \omega_{1}} \frac{\theta_{1}^{\prime}(x \mid \tau)}{\theta_{1}(x \mid \tau)}\right|_{x=\pi u / 2 \omega_{1}},
$$

where the derivative is with respect to $x$. By differentiating this relation one can obtain further identities relating the Jacobi theta functions to the Weierstrass function and its derivatives.

Another useful identity that we need:

$$
3\left[\frac{\theta_{1}^{\prime}(\pi / 3 \mid \tau)}{\theta_{1}(\pi / 3 \mid \tau)}\right]^{2}\left(\frac{\pi}{2 \omega_{1}}\right)^{2}=\wp\left(2 \omega_{1} / 3\right) .
$$




\section{B.4 "q"-expansions}

To take classical limits $(\operatorname{Im}(\tau) \rightarrow \infty)$ we use the following expansions

$$
\wp(u)=-\frac{\zeta\left(\omega_{1}\right)}{\omega_{1}}+\left(\frac{\pi}{2 \omega_{1}}\right)^{2} \operatorname{cosec}^{2} \frac{\pi u}{2 \omega_{1}}-2\left(\frac{\pi}{\omega_{1}}\right)^{2} \sum_{n=1}^{\infty} \frac{n q^{2 n}}{1-q^{2 n}} \cos \frac{n \pi u}{\omega_{1}} .
$$

Using this and $\wp(u)=-\zeta^{\prime}(u)$ and Eq.(B.12), the $q$-expansion for $\theta_{1}^{\prime} / \theta_{1}$ can be easily derived.

For evaluating classical limits of $e_{i}$, the following expansion is useful

$$
\wp(u)=\left(\frac{\pi}{2 \omega_{1}}\right)\left[-\frac{1}{3}+\sum_{n=-\infty}^{\infty} \operatorname{cosec}^{2}\left(\frac{z-2 n \omega_{2}}{2 \omega_{1}} \pi\right)-\sum_{n=-\infty}^{\infty} \operatorname{cosec}^{2} \frac{n \omega_{2}}{\omega_{1}} \pi\right] .
$$

\section{B.5 The expression of elliptic functions by means of Weierstrass functions:}

Here we quote the result in article 21.5 of [31] using Eq.(B.12) to obtain a slightly different form. Let $f(z)$ be an elliptic function with a fundamental set (i.e. modulo translations by a period) of zeros $\left(\alpha_{1}, \alpha_{2}, \ldots \alpha_{n}\right)$ and poles $\left(\beta_{1}, \beta_{2}, \ldots \beta_{n}\right)$ so that

$$
\sum_{r=1}^{n}\left(\alpha_{r}-\beta_{r}\right)=0
$$

Then

$$
f(z)=A \prod_{r=1}^{n}\left[\theta_{1}\left(\frac{\pi z-\pi \alpha_{r}}{2 \omega_{1}} \mid \tau\right) / \theta_{1}\left(\frac{\pi z-\pi \beta_{r}}{2 \omega_{1}} \mid \tau\right)\right] .
$$

where $A$ is a constant. Now if

$$
\sum_{m=1}^{m_{r}} A_{r, m}\left(z-\beta_{r}\right)^{-m}
$$

be the principal part of $f(z)$ at its pole $\beta_{r}$, then

$$
f(z)=A^{\prime}+\sum_{r=1}^{n}\left\{-A_{r, 1} \zeta\left(z-\beta_{r}\right)+\sum_{m=1}^{m_{r}-1} \frac{(-)^{m+1} A_{r, m+1}}{m !} \frac{d^{m-1}}{d z^{m-1}} \wp\left(z-\beta_{r}\right)\right\}
$$

where $A^{\prime}$ is an appropriately chosen constant.

\section{References}

[1] N. Seiberg, Nucl. Phys. B 435, 129 (1995) [arXiv:hep-th/9411149]. 
[2] I. R. Klebanov and M. J. Strassler, JHEP 0008, 052 (2000) [arXiv:hep-th/0007191].

[3] S. S. Gubser, C. P. Herzog and I. R. Klebanov, [arXiv:hep-th/0405282].

[4] D. Berenstein, JHEP 0111, 060 (2001) [arXiv:hep-th/0110184].

[5] D. Berenstein, Phys. Lett. B 552, 255 (2003) [arXiv:hep-th/0210183].

[6] D. Berenstein, JHEP 0306, 019 (2003) [arXiv:hep-th/0303033].

[7] N. Dorey, T. J. Hollowood and S. P. Kumar, JHEP 0212, 003 (2002) [arXiv:hepth/0210239].

[8] N. Dorey and S. P. Kumar, JHEP 0002, 006 (2000) [arXiv:hep-th/0001103].

[9] O. Aharony, N. Dorey and S. P. Kumar, JHEP 0006 (2000) 026 [arXiv:hepth/0006008].

[10] R. Dijkgraaf and C. Vafa, [arXiv:hep-th/0208048]

[11] R. Dijkgraaf and C. Vafa, [arXiv:hep-th/0207106]

[12] R. Dijkgraaf and C. Vafa, [arXiv:hep-th/0206255]

[13] R. G. Leigh and M. J. Strassler, Nucl. Phys. B 447, 95 (1995) [arXiv:hep-th/9503121].

[14] M. R. Douglas, [arXiv:hep-th/9807235].

[15] M. R. Douglas and B. Fiol, [arXiv:hep-th/9903031].

[16] D. Berenstein, V. Jejjala and R. G. Leigh, Nucl. Phys. B 589, 196 (2000) [arXiv:hepth/0005087].

[17] D. Berenstein, V. Jejjala and R. G. Leigh, Phys. Lett. B 493, 162 (2000) [arXiv:hepth/0006168].

[18] J. M. Maldacena and C. Nunez, Phys. Rev. Lett. 86, 588 (2001) [arXiv:hepth/0008001].

[19] F. Cachazo, K. A. Intriligator and C. Vafa, Nucl. Phys. B 603, 3 (2001) [arXiv:hepth/0103067]; F. Cachazo, B. Fiol, K. A. Intriligator, S. Katz and C. Vafa, Nucl. Phys. B 628, 3 (2002) [arXiv:hep-th/0110028].

[20] N. Dorey, T. J. Hollowood, S. Prem Kumar and A. Sinkovics, [arXiv:hep-th/0209089].

[21] N. Dorey, T. J. Hollowood, S. P. Kumar and A. Sinkovics, [arXiv:hep-th/0209099].

[22] I. K. Kostov, Nucl. Phys. B 575, 513 (2000) [arXiv:hep-th/9911023].

[23] R. Dijkgraaf and C. Vafa, [arXiv:hep-th/0302011].

[24] I. R. Klebanov and E. Witten, Nucl. Phys. B 536199 (1998) [arXiv:hep-th/9807080]. 
[25] A. Hanany and J. Walcher, JHEP 0306, 055 (2003) [arXiv:hep-th/0301231]; S. Franco, A. Hanany, Y. H. He and P. Kazakopoulos, [arXiv:hep-th/0306092]; S. Franco, A. Hanany and Y. H. He, Fortsch. Phys. 52, 540 (2004) [arXiv:hep-th/0312222].

[26] B. Fiol, JHEP 0207, 058 (2002) [arXiv:hep-th/0205155].

[27] J. Polchinski and M. J. Strassler, [arXiv:hep-th/0003136].

[28] H. M. Farkas and I. Kra, "Riemann Surfaces", Springer-Verlag (1980).

[29] T. J. Hollowood, JHEP 0310, 051 (2003) [arXiv:hep-th/0305023].

[30] M. Aganagic, R. Dijkgraaf, A. Klemm, M. Marino and C. Vafa, [arXiv:hepth/0312085].

[31] E. T. Whittaker, G. N. Watson, "A course of Modern Analysis", 4th edn., Cambridge University Press, 1927.

[32] N. Koblitz, "Introduction to Elliptic Curves and Modular Forms". Springer-Verlag, 2nd Edition 1993.

[33] S. Chiantese, A. Klemm and I. Runkel, JHEP 0403, 033 (2004) [arXiv:hepth/0311258].

[34] R. Casero and E. Trincherini, JHEP 0309, 041 (2003) [arXiv:hep-th/0304123]. 